\title{
Fusion, Magnetic Confinement
}

\author{
H.L. Berk \\ Institute for Fusion Studies \\ The University of Texas at Austin \\ Austin, Texas 78712
}

August 6, 1992

\section{DISCLAIMER}

This report was prepared as an account of work sponsored by an agency of the United States Government. Neither the United States Government nor any agency thereof, nor any of their employees "makes any warr" "ty', express or implied, or assumes any legal liability or responsibility for the accuracy, completeness, or usefuiness of any information, apparatus, product, of process disclosed, or represents that its use would not infringe privately owned rights. Reference berein to any specific commercial product, process, or service by trade name, trademark, manufacturer, or otherwise does not necessarily constitute or imply its endorsement, recom. mendation, or favoring by the United States (jovernment or any agency thereof. The viewr and opinions of authors expressed herein do not necessarily state or reflect those of the United States Government or any agency thereof. 


\section{Abstract: Fusion, Magnetic Confinement H.L. Berk}

An overview is presented of the principles of magnetic confinement of plasmas for the purpose of achieving controlled fusion conditions. Sec. 1 discusses the different nuclear fusion reactions which can be exploited in prospective fusion reactors and explains why special technologies need to be developed for the supply of tritium or ${ }^{3} \mathrm{He}$, the probable fuels.

In Sec, 2 the Lawson condition, a criterion that is a measure of the quality of confinement relative to achieving fusion conditions, is explained. In addition, an overview is given of the principal energy loss mechanisms that are present in a magnetically confined plasma.

In Sec. 3 fluid equations are used to describe plasma confinement. Specific confinement configurations are considered. The first examples given are for azimuthally symmetric pinches, which are of infinite extent in the $z$-direction but confined in the racial direction. These solutions are shown to be paradigms for the tokamak, the reversed field pinch as well as the open-ended mirror machine. The tokamak configuration is then discussed in toroidal geometry, where it is noted that a current drive mechanism, as well as a vertical field to prevent plasma expansion, needs to be provided. In addition, compact toroids and stellarator configurations are discussed.

In Sec. $t$ the orbits of particles in magnetic and electric fields are discussed. It is noted that collisions prevent perfect confinement, and estimates 
are made for the rate energy is iost by collisional processes. In open-ended mirror machines, the power losses due to collisions are comparable to the power that can be produced by the fusion reaction. This difficulty has lead to the development of the tandem mirror concept, where electric fields, together with magnetic fields, are used to improve energy confinement. For tokamaks it is shown that orbital excursion from magnetic flux surfaces, together with collisions, leads to so-called neochassical diffusion, where transport losses are significantly larger than would be estimated from the cross-field transport of a plasma in a uniform magnetic field. Simple physical arguments are given to estimate neoclassical transport coefficients. One of the most important transport mechanisms is the bootstrap current where it is found that the radial pressure gradient of a tokamak can by itself generate a significant fraction of the required tokamak current.

In Sec. 5 stability considerations are discussed. It is noted that confinement systems usually need to satisfy stability constraints imposed by ideal magnetohydrodynamic (MHD) theory. Otherwise, rapid disruption of the plasma configuration is likely. As a result, the most favorable configurations are not determined by the highest values of kinetic pressure (for fixed magnetic pressure) that can be achieved in equilibrium theory, but by the highest values of kinetic pressure that can be achieved on the basis of stability theory. Some of the stability constraints imposed by .MHD stability theory may be significantly altered by having energetic particles in the system and some 
proposed examples are given. A brief discussion is given of microinstabilities, which are short wavelength oscillations that are responsible for so-called anomalous transport, a crucial aspect of plasma confinement.

The paper culminates with a summary of experimental progress in magnetic confinement. Present experiments in tokamaks have reached the point that the conditions necessary to achieve fusion are being satisfied. 


\section{Contents}

1. ENERGY FROM THE FUSION REACTION ....... 5

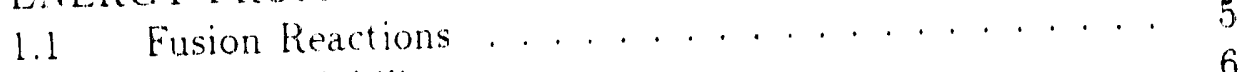

$1.2 \quad$ Fuel Availability . . . . . . . . . . . . . 6

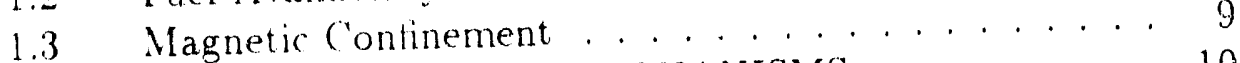

2. INTRINSIC ENERGY LOSS MECHANISMS . . . . . . 10

2.1 Lawson Criterion . . . . . . . . . . . . 10

2.2 Loss Mechanisms .................. 12

2.2 .1 Bremsstrahlung............. 12

2.2 .2 Synchrotron radiation . . . . . . . . 13

2.2 .3 Diffusion ............... 14

2.2 .4 Turbulence losses . . . . . . . . . 15

3. MAGNETICALLY CONFINED EQUILIBRIUM ..... 16

3.1 Magnetohydrodynamic Equilibrium Equations . . . . 17

3.2 Two-Dimensional Pinch Solutions . . . . . . . . 18

3.3 End Stoppering . . . . . . . . . . . . . . . 21

3.4 Toroidal Configurations . . . . . . . . . . . 22

3.4 .1 General considerations .......... 22

3.4 .2 Vertical field ............... 23

3.4 .3 Compact toroids ............24

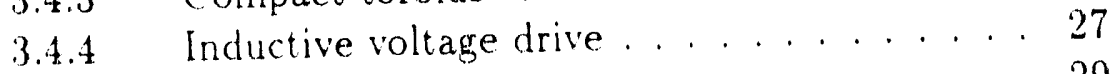

3.4.5 Stellarators .................... 29

4. PARTICLE ORBITS AND CLASSICAL TRANSPORT IN

MAGNETICALLY (ONFINED PLASMAS . . . . . . . 31

4.1 Particle Orbits in Magnetic and Electric Fields ..... 31

4.2 Spatially Varying Fields ... . . . . . . . . 34

$+2.1 \quad Q$-value in mirror machine ........ 37 
4.2.2 Electrostatic potentials in tandem mirrors . . 38

4.3 Orbits in Toroidal Field . . . . . . . . . . . . . . . 39

4.4 Nexclassical Diffusion ................ 43

4.41 Bootstrap current ............ 45

4.4 Ware pinch .............. 46

4.5 Raclial Electric Field . . . . . . . . . . . . 47

4.6 Orbits in Stellarators .............. . . 48

5. PLASMA INSTABILITY AND ANOMALOUS TRANSPORT 50

5.1 Ideal MHD Theory ............... 50

5.1 .1 Simplified dynamical model ....... . 50

5.1 .2 Generalization to plasmas . . . . . . . . . 52

5.1 .3 Role of field line curvature . . . . . . . . 53

5.2 Deviation from MHD stability guidelines . . . . . . 54

5.2 .1 Passive current carriers . . . . . . . . 55

5.2 .2 Finite Larmor radius stabilization . . . . . . 55

5.2 .3 Migma concept ............ 56

5.3 Stability in Tori ............... 56

5.3 .1 Line average stabilization ........ 57

5.3 .2 Beta stability limit ........... 57

5.3 .3 RFP stability ............ 58

5.4 Resistive Stability . . . . . . . . . . . . 58

5.5 Microinstability ............... 60

5.5 .1 Velocity space instability......... 61

5.5 .2 Drift wave instability . . . . . . . . 62

5.6 Turbulent Diffusion ............... . 63

6. STATUS OF MAGNETIC FUSION RESEARCH . . . . . 64

6.1 Historical Overview . . . . . . . . . . . . 64

6.2 Status of Non-Tokamak Concepts . . . . . . . . 66

6.2 .1 Mirror machines ...........66

6.2 .2 Reversed field pinch . . . . . . . . . 68

6.2.3 Compact toroids . . . . . . . . . . 69

6.2 .4 Stellarators ............... 71

6.3 Status of Tokamak Research . . . . . . . . . . 72

6.3 .1 Impurity control and fueling . . . . . . . 73

6.3 .2 Auxiliary heating . . . . . . . . . . 73

6.3.3 Plasma shaping ............. 74

6.3.t Current drive .............. . . . . . . . 
6.3.5 Achieved confinement parameters . . . . . 76

6.4 Conclusion . . . . . . . . . . . . . . . . . 


\section{INTRODUCTION}

Nuclear fusion is the combining of light nuclei to form more massive ones with the conversion of nuclear binding energy to kinetic energy. This energy conversion process is the source of heat in the stars and in the Sun. If this reaction can be replicated on Earth in a controlled manner, there would exist an energy source to supply mankind in perpetuity.

In order to achieve the fusion reaction, charged nuclei have to approach to within distances of $10^{-12} \mathrm{~cm}$ of each other for nuclear transitions to occur. However, this close approach is impeded by the Coulomb repulsive force of the positively charged nuclei. Only if particles have enough kinetic energy, so that they can penetrate this Coulomb barrier, can the fusion reaction take place. Hence, to achieve fusion it is necessary to heat the fuel to a sufficiently high temperature so that particles can penetrate close to each other's nuclear core. Then a nuclear fusion environment can be created where the kinetic energy released by the fusion reaction products maintains the temperature of the fuel.

The fusion environment is at a temperature even greater than that found in the Sun. In order to achieve this environment technologically, special conditions are required to establish insulation of the fuel from the surrounding "cold" Earth temperature. Otherwise the energy losses to the neighboring environment will exceed the energy produced by the fusion reaction. The task of producing adequate heat confinement is the crucial aspect of the con- 
trolled fusion program. As will be described here, great progress has occurred since the program initiated in the $1950 \mathrm{~s}$, yet much more is needed in order to achieve one of the great challenges to mankind; Earth-based energy from controlled fusion.

\section{ENERGY FROM THE FUSION REACTION}

Only a few fusion reactions have sufficiently high rates and energy gain to be exploitable in a practical fusion reactor. As a result the issue of what fuels are available for use on Earth when controlled fusion is feasible, is significant and is discussed in this section.

\subsection{Fusion Reactions}

The three most important fusion reactions for energy applications are

$$
\begin{aligned}
& d+d \rightarrow\left\{\begin{array}{l}
{ }^{3} \mathrm{He}+n+3.3 \mathrm{MeV} \\
\mathrm{t}+p+4.0 \mathrm{MeV}
\end{array}\right. \\
& \mathrm{d}+\mathrm{t} \rightarrow{ }^{4} \mathrm{He}+n+17.6 \mathrm{MeV} \\
& \mathrm{d}+{ }^{3} \mathrm{He} \rightarrow{ }^{4} \mathrm{He}+p+18.3 \mathrm{MeV} .
\end{aligned}
$$

Figure 1 shows the cross-section and reaction rates of these three reactions. These curves show that the energy dependence of the rate of fusion reactions decreases rapidly below the energy of maximum reactivity, a manifestation that the Coulomb barrier can quench the fusion reaction. A fusion environment must achieve temperatures in excess of the ideal ignition temperature, 
$7_{\text {ideal }}$, which is the lowest temperature for which a self-sustaining fusion reaction can be achieved when energy losses only due to bremsstrahlung radiation are accounted for. For the $\mathrm{d}-\mathrm{t}$ reaction $T_{\text {ideal }}$ is $5 \times 10^{7}$ kelvin, or in units more commonly used by research workers, 4 kilo electron volts (kev). For the d-d and $\mathrm{d}^{3}{ }^{3} \mathrm{He}$ reactions the ideal temperatures are 30 and $40 \mathrm{keV}$ respectively.

\subsection{Fuel Availability}

One of the principal motivations for using controlled fusion for energy is the availability of fuel reserves. Among the reactions listed in Fig. 1, the d-d reaction clearly is a fuel rescurce with virtually inexhaustible supply. Deuterium is a stable isotope of hydrogen, which is stored in sea water with a natural abundance of 1 part in 6500. The energy content of 1 liter of water from the $d-d$ reaction $\left(3 \times 10^{9}\right.$ joules $)$ is equivalent to the energy content of about 100 liters of oil. The effective energy content can even be raised substantially if the tritium and ${ }^{3} \mathrm{He}$ products produce further fusion reactions. With deuterium extracted from sea water, if energy is consumed at a rate of $10^{21}$ jonles/year, the $d$ - $d$ reaction can provide an energy source for $\sim 10^{10}$ years.

The main drawback of the $d$-d reaction is a relatively low reaction rate and yield, which makes its implementation more difficult as compared to the $\mathrm{d}-\mathrm{t}$ and $\mathrm{d}-{ }^{3} \mathrm{He}$ reactions. Of the two, $\mathrm{d}-\mathrm{t}$ has a clear advantage in achieving a fusion environment, as the ignition temperature is appreciably lower and 
reaction rates are higher than the $\mathrm{d}-{ }^{3} \mathrm{He}$ reaction. For this reason the first planned demonstration of controlled fusion conditions is based on the $\mathrm{d}$-t reaction.

As a long term energy source, the $\mathrm{d}$-t reaction is feasible, although not as directly as with the $d-d$ reaction. Tritium is an unstable element with a half-life of 12 years. Hence tritium must be constantly bred during reactor operation. Breeding is possible via the nuclear reactions

$$
\begin{aligned}
& n+{ }^{6} \mathrm{Li} \rightarrow \mathrm{t}+{ }^{4} \mathrm{He}+4.8 \mathrm{MeV} \\
& n+{ }^{7} \mathrm{Li} \rightarrow \mathrm{t}+{ }^{4} \mathrm{He}+n-2.5 \mathrm{MeV} .
\end{aligned}
$$

Both reactions can be used to produce tritium. The $n-{ }^{6} \mathrm{Li}$ reaction is exothermic and if the released energy is exploited, the effective yield per d-t reaction would be $21 \mathrm{MeV}$. Though the ${ }^{7} \mathrm{Li}$ reaction is endothermic, it does not appreciably degrade the energy output of the entire d-t cycle. ${ }^{6} \mathrm{Li}$ has a natural abundance of $7.5 \%$ while ${ }^{7} \mathrm{Li}$ has a natural abundance of $92.5 \%$. Thus as tritium will ultimately be bred from lithium, it is the availability of lithium that governs the energy potential of the $d-t$ reaction. The officially estimated "economically" extractable lithium reserves are equivalent to about one thousand years of energy supply. Lithium is also found in sea water, which if economically extractable, would imply an energy reserve on the scale of millions of years.

Despite its long term potential, the implementation of fusion energy based on $d-t$ has several difficulties. The need to breed tritium complicates reactor design and since it is radioactive, there will be siting restrictions. There 
is controversy about whether the hazards, associated with the long lived radicactive material generated from the neutrons produced by the $d$-t reaction, are a significant environmental concern (though it is generally agreed that such problems are far less severe than with fission reactors). Perhaps the most significant problem is whether the long term integrity of containment vessels can be maintained under the continuous flux of $14 \mathrm{MeV}$ neutrons (advarced materials research is needed to demonstrate that walls can be fabricated from material that anneals with minimum deterioration from defects caused by neutron bombardment).

These concerns give impetus to the desire to exploit the $\mathrm{d} \cdot{ }^{3} \mathrm{He}$ fusion reaction, which by itself produces only charged fusion products (though some neutrons are produced from the $\mathrm{d}-\mathrm{d}$ side reaction). Another potential benefit of the $\mathrm{d}-{ }^{3}$ He reaction is the possibility of directly converting charged fusion products to electrical energy with an efficiency of $\sim 80 \%$, whereas energy derived from energetic neutrons needs first to be converted to heat, so that the ultimate conversion to electricity is limited to a thermodynamic energy efficiency of $\lesssim 40 \%$.

Unfortunately there is crucial uncertainty in the $\mathrm{d}-{ }^{3} \mathrm{He}$ cycle which is that of fuel availability. Though ${ }^{3} \mathrm{He}$ is a stable element, it is light enough to escape the Earth's gravitational field. Hence only trace amounts of ${ }^{3} \mathrm{He}$ are found on Earth. Paradoxically, ${ }^{3} \mathrm{He}$ is present on the moon [Wittenberg, et al., 1986]. The ${ }^{3} \mathrm{He}$, which originates in the flux of the solar wind, is embedded in lunar 
rock. It has been estimated that this ${ }^{3}$ He source can supply mankind with energy for hundreds of years. On a longer time scale. one can envision space travel to the larger planets. Jupiter and Saturn, where the gravitational field is strong enough to retain "He. An alternative scenario is that. Earth-based sources of ${ }^{3}$ He can come from other fusion reactors operating on $\mathrm{d}-\mathrm{d}$ or $\mathrm{d}$-t reactions. Veutron proflucing reactors could be sited in remote areas and supply ${ }^{3} \mathrm{He}$ for electricity producing ${ }^{3} \mathrm{He}$ reactors close to populated areas.

The above discussion indicates that with appropriate technological progress, the fusion reaction from several fuels can provide civilization with a long term energy source. The choice of fuel cycle will depend on the quality of the energy confinement scheme that is devised, the environmental standards that are deemed acceptable. and the technologies that are developed in the future.

\subsection{Magnetic Confinement}

Still. the first major step remains to be proved: i.e. can a controlled fusion Earth-based environment be established? There has been considerable progress towards answering this question, but much more information is needed from future research. This article is devoted to explaining the principles in magnetic confinement, where an ionized gas (commorily called plasmas) is isolated from the surrounding "cold" environment by magnetic pressure.

In the subsequent sections we present the following material: In Sec. 2 
we discuss the confinement parameters that must be achieved if the energy produced from the fision reaction is to exceed the energy required to produce the hot plasma conditions necessary for copious fusion reactions to occur. In addition we will present an overview of the principal energy loss mechanisms associated with a confined hot plasma. In Sec. 3 we discuss magnetically confined equilibrium and give an example several confinement schemes. In Sec. \& we examine in more detail the mechanisms for particle confinement and transport for two of the most important confinement concepts, the mirror machine and the tokamak. The viability of a plasma confinement concept usually deper.ds on the stability of the configuration. An overview of stability considerations is given in Sec. 5. Finally, in Sec. 6 past progress and the present states of magnetic confinement research is discussed.

\section{INTRINSIC ENERGY LOSS MECHANISMS}

\subsection{Lawson Criterion}

All fusion confinement schemes rely on heating a plasma in a configuration that is sufficiently well insulated from its surroundings. The Lawson criterion, the product of the particle density $n$ and energy lifetime $T$ arising from all possible loss mechanisms, is a measure of how close a given configuration is to creating a fusion environment. It is obtained by observing that a plasma (with say equal number of electrons and hydrogenic ions) with a temperature $T$ (throughoun Sec. 2 we assume electron and ion temperatures 
are equal), electron density $n$, and lifetime $\tau$, loses power density $P_{l}$, at a rate $P_{l}=3 n k T / \tau$. In order to have a self-sustaining fusion reaction, this power loss must be compensated by the energy produced by the fusion reaction. It is envisioned that charged fusion products will be contained in the system with their kinetic energy being absorbed by the background plasma, while fusion produced neutrons escape the plasma region. A self-sustaining reaction (a burn) occurs when the pover production of the charged particle fusion products compensates the thermal energy !nses of the plasma. Thus the criterion for a fusion burn is

$$
\frac{3, i k T}{T}-\leqslant n^{2}\langle\sigma v\rangle W_{F}
$$

where $\langle\sigma v\rangle$ is the fusion reaction rate averaged over the background ion distribution functions ( $\sigma$ is the fusion cross section, $v \equiv$ relative ion velocities, and $\gamma$ a numerical factor that depends on the fuel mixture (for $d$-d, $\gamma=1 / 2$, for $d-t, \gamma=n_{d} n_{t} / n^{2} \leq 1 / 4$, etc.; the subscripts $d$ and $t$ refer to deuterium and tritium and $W_{F}$ the energy of the charged fusion products. From Eq. (1) the Lawson criterion follows

$$
n \tau>\frac{3 k T}{i\langle\sigma v) W_{F}} \equiv(n T)_{L}
$$

where the right-hand side depends on temperature and fuel mix. Figures of nT v's temperature, for the optimum fuel mix is given in Fig. 2. One observes that $n t$ values in excess of $10^{20} \mathrm{~m}^{-3} \mathrm{~s}$ is needed to achieve self-sustaining fusion. 
A less stringent, modified Lawson criterion applies to the condition for obtaining net energy when energetic neutrons are produced. For the $d-t$ fuel cycle with ${ }^{6} \mathrm{Li}$ used for the breeding of tritium, $22 \mathrm{MeV}$ per reaction is released, with only $3.5 \mathrm{MeV}$ going into charged products inside the plasma. With a $40 \%$ heat conversion efficiency, the critical $n T$ that is needed to achieve fusion energy is reduced by a factor of 2.5. However, external power must then be continuously supplied to this so-called "wet-wood burner" mode of operation.

\subsection{Loss Mechanisms}

The feasibility of attaining fusion energy depends on devising thermal insulation so that Eq. (2) can be satisfied. There are several intrinsic power loss mechanisms that place upper bounds on $n \tau$ including: radiation, diffusive losses due to collisions, convection due to disassembly of the equilibrium, and diffusion due to small-scale turbulence. The composite loss rate can be written as $1 / T=\Sigma_{1} 1 / T_{i}$, with $T_{i}^{-1}$ ( the effective energy loss rate per electron-ion pair) for each individual mechanism.

\subsubsection{Bremsstrahlung}

Radiation due to bremsstrahlung is a basic loss mechanism and is due tc the radiation emitted by electrons colliding with themselves and with ions. The 
power radiated, $P_{\text {brem, }}$, is given by

$$
P_{\text {brem }}=\frac{3 n k T}{\tau_{\text {brem }}}
$$

with $n T_{\text {brem }}=2.8 \times 10^{21} T_{10 k}^{1 / 2} / Z_{\text {eff }} m^{-3} s$ where $T_{10 k}$ is the plasma temperature in units of $10 \mathrm{keV}$ and $Z_{\mathrm{eff}}=\sum_{1} Z_{i}^{2} n_{i} / n_{e}$ with $Z_{i}$ the atomic number of species $i$. The subscript $\epsilon$ refers to electrons. As bremsstrahlung is not significantly reabsorbed by the plasma in configurations appropriate to controlled fusion, the losses are not readily "recycled" back into the system (except perhaps with some thermodynamic efficiency $\sim 35-40 \%)$. Impurities sputtered from the wall can accumulate in plasmas and can cause significant increase in the bremsstrahlung loss rates even if the hydrogen component is not suffciently diminished. Hence the suppression of impurity accumulation is critical in plasma containment. Because of bremsstrahlung losses many possible neutron-free fusion reactions cannot be used for fusion power production. Examples include: $p+{ }^{11} \mathrm{~B} \rightarrow 3 \alpha+8.7 \mathrm{MeV}, p+{ }^{6} \mathrm{Li} \rightarrow{ }^{4} \mathrm{He}+{ }^{3} \mathrm{He}+4 \mathrm{MeV}$, etc.

\subsubsection{Synchrotron radiation}

The other important radiation process with significant energy loss is synchrotron radiation. This is single particle radiated power due to cyclotron motion of electrons in a magnetic field which is given by

$$
P_{\mathrm{sync}}=\frac{3 n T}{T_{\mathrm{sync}}}
$$


with

$$
n T_{\text {sync }}=\frac{.97 \times 10^{21}, 3 \mathrm{~m}^{-3} \mathrm{~s}}{T_{10 k}}
$$

with $3=4 \mu_{0} k T / B^{2}$ the ratio of kinetic pressure to magnetic pressure, a fundamental plasma parameter in magnetic fusion. If the radiated power would propagate without further absorption, this parasatic loss mechanism would severely effect the viability of nearly every mannetic fusion concept. Fortunately, most of the radiation is reabsorbed by the plasma, and even escaping radiation can be reflected by external conducting walls and reabsorbed by the plasma. Calculations show that synchrotron losses is not a constraining loss mechanism in a $\mathrm{d}$ - $t$ reactor, whereas they do constrain $\mathrm{d}-\mathrm{d}$ or $\mathrm{d}-{ }^{3} \mathrm{He}$ reactors to operate at moderately high $\beta$ values (typically $\beta \gtrsim 15 \%$ ).

\subsubsection{Diffusion}

The other important mechanism that determines plasma lifetime is particle diffusion. In subsequent sections we shall describe magnetic containment configurations in which the particle orbits are isolated from the surrounding walls. However, binary collisions and fluctuating fields cause particles to deviate from their orbits, and eventually these particles diffuse to the walls. Energy losses due to binary collisions can be reliably calculated. Such losses, together with radiation. stablish the intrinsic containment properties of a given configuration. In toroidally shaped devices collisional losses are generally compatible with reactor operation. In open-ended devices, these losses 
are a significant constraint for power production.

\subsubsection{Turbulence losses}

Losses due to fluctuations from instabilities appear to govern the lifetime of almost every magnetic fusion experiment that has been developed. Roughly, these losses can be categorized in two forms, those due to macroinstability and those due to microinstability. In macroinstability, the unstable mode affects the entire plasma column and the loss of integrity of the confinement scheme. In such cases the losses are usually incompatible with achieving energy production. However, in some cases macroinstability is localized to the plasma interior, and the integrity of the thermal insulation can still be maintained.

In microinstability the instability structure is on a small scale compared to the plasma size. These fluctuations cause stochastic motion of particles, much like that produced by collisional diffusion. Typically it is this diffusive process that appears to determine thermal containment time of even the best contained plasmas. The detailed mechanisms for such losses are not fully understood, and the theoretical and empirical description of these losses are still being established [Callen, 1992].

The control of these self-induced fluctuations is a key to obtaining the plasma parameters needed to fulfill the Lawson condition. Such fluctuations can have a beneficial effect. For example they can prevent the build-up of unwarranted impurity in the plasma center or they can allow dynamo action 
to steadily maintain the magnetic structure of a configuration that would not be possible to sustain in absence of fluctuations. It now appears that at least in tokamak experiments, that the natural fluctuation level is compatible with the achievement of controlled fusion conditions.

\section{MAGNETICALLY CONFINED EQUILIBRIUM}

In order to prevent heat from escaping from a confined hot plasma to the cold surroundings, a means of providing both confinement forces and thermal insulation is needed. Magnetic fields can in principle provide both properties.

Many different magnetic field configurations have been proposed. Roughly the proposals can be divided into two classes, toroidal and open-ended. In the former case the trajectories of magnetic field lines lie on a surface of a torus, as shown in the spiral lines in Fig. 3. Charged particles, which constitute the plasma, move nearly parallel to these field lines, and as a result under many conditions the particles remain inside the torus. In open-ended machines the trajectories of magnetic field lines inside the plasma reach the surrounding wall as shown in the two configurations of Fig. 4. However, particles are inhibited from reaching the wall if the magnetic field strength increases toward the wail.

In this section we will discuss some basic ideas in magnetic plasma containment and show how they have been specifically applied to various containment concepts. 


\subsection{Magnetohydrodynamic Equilibrium Equations}

To understand containment one can examine solutions of the magnetohydrodynamic (MHD) equilibrium equations, which take the form

$$
\begin{aligned}
& \mathbf{j} \times \mathbf{B}=\boldsymbol{\nabla} \cdot \mathbb{P} \\
& \boldsymbol{\nabla} \times \mathbf{B}=\mu_{0} \mathbf{J}
\end{aligned}
$$

where $\mathbf{B}$ is the magnetic field. $\mathbf{j}$ the current density, and $\mathbb{P}$ the generalized momentum flux ten sor. It is frequently appropriate to take $\mathbb{P}$ as isotropic, whereupon $\mathbb{P}=p \mathbf{I}$ with $p$ the scalar pressure and $\mathbf{I}$ the unit dyadic (pressure isotropy is established if the particle lifetime is longer than the collisional relaxation time to a Maxwellian distribution). These equations can be written in stress conservation form,

$$
\boldsymbol{\nabla} \cdot \boldsymbol{\Pi}=0
$$

with the stress tensor given by, $\Pi=\frac{\mathbf{B B}}{\mu_{0}}-\frac{B^{2}}{2 \mu_{0}} \mathbf{I}-\mathbb{P}$. One can show from the virial theorem that Eq. (5) only allows solutions if on a surface, $S$, bounding the containment volume, the "force," $\int \boldsymbol{\Pi} \cdot \mathbf{n} d S$ is nonzero, with $\mathbf{n}$ the unit normal vector to the bounding surface $S$. This means that in order to obtain equilibrium one must supply an external anchor. One type would simply be plasma leaning against external solid material walls. This method is not generally satisfactory since thermal heat losses would usually be too large (nonetheless there are serious "wall containment" concepts, such as originally proposed by Budker, that are being developed that depend upon the relatively low heat conduction across magnetic fields to obtain the needed 
thermal insulation (Ryutov, 1975]). The more standard technique is to have magnetic coils, which establish external magnetic fields that can isolate the plasma from wall contact. In turn these coils need to be anchored by external forces.

\subsection{Two-Dimensional Pinch Solutions}

To begin to understand the solutions of Eq. (5), it is informative to consider a system that is unbounded in the $z$-direction, symmetric in the $\theta$-direction (where $\theta$ is the angle about the $z$-axis), and contained radially in $r$. If we take the momentum flux tensor $\mathbf{P}$ as isotropic, $\mathbb{P}=\mathbf{p} \mathbf{I}$ (note $p$ is the particle pressure) and the magnetic field of the form $\mathbf{B}=\hat{\mathbf{z}} B_{z}(r)+\hat{\boldsymbol{\theta}} B_{\theta}(r)$, the general solution to Eq. (5) is

$$
p(r)+\frac{B_{z}^{2}(r)}{2 \mu_{0}}-\left\langle p+\frac{B_{z}^{2}}{2 \mu_{0}}\right\rangle=\frac{B_{\theta}^{2}(r)}{2 \mu_{0}}
$$

where $\langle a(r)\rangle=2 r^{-2} \int_{0}^{r} d r^{\prime} r^{\prime} a\left(r^{\prime}\right)$.

There are several issues that this form illustrates. First of all, at a wall $r=a$, the pressure, $p(\alpha)$, can vanish, and the averaged contained pressure is then

$$
\langle p\rangle=\frac{B_{z}^{2}(a)+B_{\theta}^{2}(a)-\left\langle B_{z}^{2}\right\rangle}{2 \mu_{0}} .
$$

We see that the particle pressure can be expressed in terms of a containment parameter,

$$
\langle\beta\rangle=\frac{2 \mu_{0}\langle p\rangle}{B_{z}^{2}(a)}
$$


where $B_{z}(a)$ is also the applied external magnetic field if there are no embedded external current carriers inside the plasma. Equation (7) then becomes

$$
\langle\beta\rangle=1-\left\langle b_{z}^{2}\right\rangle+b_{\theta}^{2}(a)
$$

with $b_{j}(r)=B_{\jmath}(r) / B_{z}(a)$.

We now note that there is freedom in specifying $b_{z}$ or $b_{\theta}$. The case where $b_{\theta}=0$ is the "theta pinch" solution, where the nomenclature arises because in such a configuration the plasma current is in the $\theta$-direction. In this case one can show that $1>b_{z}^{2}(r)$, hence $\langle\beta\rangle \leq 1$. This is why $\beta$ is frequently used as a measure of effectiveness of stored plasma pressure with respect to an applied magnetic field.

In principle the greatest effectiveness in confining plasma is obtained with the $z$-pinch solution where plasma currents are in the $z$-direction and $B_{z}=0$. This case does not require any external magnetic field to achieve radial containment (note that the $\theta$-pinch is unbounded in the $z$-direction, which is why the virial theorem is not violated). Thus from just its equilibrium properties, the $z$-pinch is extremely attractive for fusion application. Unfortunately, in its most basic form the $z$-pinch is highly unstable and drastic modifications of the configuration is usually considered necessary to have a tractable concept. Nonetheless, interest in the pure $z$-pinch continues today. Novel ideas for stabilization are being investigated by Lehnert in the Ex-trap experiment [Drake, et al., 1989]. There is also considerable interest by several groups in the high density fast z-pinch produced from thin fibers, e.g. [Haines, et al., 
1991], where the time scale for producing fusion energy might be more rapid than the time scale for instability to develop.

The most direct way to stabilize a $z$-pinch is to have simultaneously both $\hat{z}$-directed and $\hat{\theta}$-directed curre"ts. In Eq. (7) the kinetic pressure and the magnetic pressure due t.c are completely interchangeable. In the limit of zero kinetic pressure, the solution is a "force-free solution" where the plasma currents are parallel to the magnetic field so that the magnetic force, $\mathbf{j} \times \mathbf{B}=0$. There is a class of solutions, where the direction of $B_{z}$ changes inside the discharge, and such solutions are characteristic of the Field Reversed Pinch (RFP), one of the important, ncepts in controlled fusion research.

Another prototype solution contained in the structure of to Eq. (7) is where $B_{z}$ is nearly constant within the discharge and the plasma beta is low. Typically, $\beta_{z}=2 \mu_{0} p / B_{z}^{2} \ll 1$ while $\beta_{\theta}=2 \mu_{0} p / B_{\theta}^{2} \approx 1$. Such discharges are stable for a wide range of parameters and are the basis of the so-called tokamak, the lead concept for achieving fusion by magnetic containment. Many issues in tokamak containment will be discussed later.

Both these "low- $\beta$ " solutions sacrifice high- $\beta$ effectiveness in exchange for achieving stability. Beta values $\sim 10 \%$ have been achieved in these concepts and the determination of the upper permissible beta is one of the important questions under investigations in controlled fusion. 


\subsection{End Stoppering}

The MHD solutions presented thus far do not exhibit axial containment. For axial containment we either have to "stopper" the ends or wrap the straight solutions into a torus. First we discuss end stoppering.

Axial stoppering can be achieved by varying the magnitude of the magnetic field along the $\bar{z}$-axis if the pressure tensor is anisotropic. As particle orbits in a magnetic field causes the pressure tensor to be symmetric about the magnetic field direction, while the parallel component is unaffected by the magnetic field, the pressure tensor $\mathbb{P}$, takes the form

$$
\mathbb{P}=p_{i L}(\mathbf{I}-\mathbf{b} \mathbf{b})+p_{\|} \mathbf{b} \mathbf{b}
$$

where $\mathbf{b}$ is in the direction of the magnetic field, $p_{\perp}$ the perpendicular pressure and $p_{\|}$the parallel pressure. The component of Eq. (11) along the magnetic field gives the relation,

$$
\frac{\partial p_{\|}}{\partial s}=\frac{-\left(p_{\perp}-p_{\|}\right)}{B} \frac{\partial B}{\partial s}
$$

where $s$ is the distance along a field line. This equation allows the pressure $p_{\|}$to be contained along the magnetic field if $B$ increases towards the ends. This axial containment is an example of the mirror principle. In Sec. 4 we will study in more detail how electric and magnetic forces act on particles to achieve containment. 


\subsection{Toroidal Configurations}

The two-dimensional pinch solution is frequently considered as a mathematical limit of a large aspect ratio toroidal system. However this "attachment" of ends of a nearly straight system onto itself, hrings in many new physical features, some of which is discussed in this section and others in Sec. 4.

\subsubsection{General considerations}

To study containment in toroidal configurations, Eq. (5) has to be solved in three-dimensional geometry. A typical three-dimensional configuration is shown in Fig. 3. In such a configuration there is a toroidal axis of radius $R_{0}$ (the dashed curve in Fig. 3) on which a magnetic field closes on itself. Frequently $R_{0}$ is constant, but in principle $R_{0}$ can vary as it does in some concepts. The other field lines wrap around the toroidal axis as indicated by the lightly drawn spirals in Fig. 3. The winding number of a field line is the ratio of the number of times it wraps around poloidally (the short way around) to the number of times it wraps around toroidally (the long way around). The winding number is commonly called the rotational transform $\iota$, and its inverse, $1 / \iota$, is denoted as $q$, the safety factor, which is a parameter closely related to plasma stability. A convenient set of coordinates, shown in Fig. 3 , is the toroidal angle $\phi$, the poloidal angle $\theta$, and the radial distance to the magnetic axis $r$. For a toroidally symmetric configuration one can readily show that if the rotational transform is irrational, a single field line 
is constrained to lie on a surface. Such a "magnetic flux" surfaces can also be attained in configurations that are not axisymmetric. This property leads to the stellara:or concept, to be discussed below, where the flux structure can be formed directly from external magnetic coils, rather than the internal plasma current.

\subsubsection{Vertical field}

In a toroidal pinch, there will be a toroidal plasma current, $I_{p} \bar{\phi}$ that produces $B_{\theta}$ fields. The average self-magnetic force, $\left\langle\mathbf{I}_{p} \times \mathbf{B}_{i s}\right\rangle$ with $\mathbf{B}$, the magnetic field produced by $\mathbf{I}_{p}$, produces the so-called hoop force directed outward in the $\widehat{\mathbf{R}}$-direction. This self-force tends to produce expansion of the current ring. To obtain confinement, this force must be compensated by an external vertical magnetic $\mathbf{B}_{v}$, directed such that $I_{p} \widehat{\phi} \times \mathbf{B}_{v}$ is in the $-\widehat{\mathbf{R}}$-direction. For a torus of large aspect the magnitude of $\mathbf{B}_{v}$ is found to be

$$
\mathrm{B}_{v}=\frac{a B_{\theta}(a)}{2 R_{0}}\left(\ln \frac{8 R_{0}}{a}-\frac{3}{2}+\frac{\ell_{i}}{2}+\left\langle\beta_{\theta}\right\rangle\right)
$$

where $B_{\theta}(a)$ is the poloidal component of the magnetic field that is generated by the plasma current, $I_{p} \approx \frac{2 \pi a B_{\theta}(a)}{\mu_{0}},\left\langle\beta_{\theta}\right\rangle=2 \mu_{0} \int_{0}^{a} d r r p(r) / a^{2} B_{\theta}^{2}(a)$ is the average beta with respect to the magnetic field and $\ell_{i}$ (the internal inductance) is a quantity of order unity that depends on the detailed current profile. The need of such an external vertical field can be viewed as a consequence of the virial theorem.

From Eq. (13) one infers that the intrinsic 3 -value with respect to the 
vertical field is large with $3 \approx R / a$. Such high beta values can arise if the toroidal magnetic field, $B_{\infty}$, is zero. However, stability considerations demand the presence of an external toroidal magnetic field, $B_{\phi}$, which considerably reduces the $\beta$ that can be achieved. In the RFP the toroidal $B_{\phi}$, and poloidal, $B_{\theta}$, magnetic fields are comparable in magnitude, while in the tokamak $B_{\phi}$ is considerably larger than $B_{\theta}$, typically $B_{\phi} / B_{\theta} \approx R / a$.

We also note that toroidal magnetic fields are typically produced by magnetic field coils where the coils are wound through the hole in the plasma as shown schematically in Fig. 5a. In Fig. 5b the plasma region and toroidal field coil is shown as it exists in the Text tokamak. The inner coil structure gives rise to complications that would be worthwhile to avoid if an alternate configuration proved suitable. This is a motivation for the study of compact toroids where there are no external coils through the plasma hole.

\subsubsection{Compact toroids}

As $3 \approx a / R$ appears to be a limitation of confinement, increased $\beta$-values is achieved by increasing $a / R$. The extreme is to have $a / R \approx 1$. If in this case one eliminates the external toroidal field coils entirely, and one need not have hardware through the inside of the torus. Instead, the plasma can reach the axis of the machine, and one has a configuration known as a compact toroid.

To discuss the structure of the magnetic fields in a compact toroid, we 
introduce a magnetic flux function $w_{p}$ where

$$
\mathbf{B}_{\theta}=\frac{\nabla \psi_{p} \times \hat{\phi}}{2 \pi R}
$$

The magnetic field then lies along contours of constant $\psi_{p}$. A schematic figure of the flux surface of a compact toroid is shown in Fig. 6. The $z$-axis is an axis of revolution. The poloidal magnetic field is closed and reverses direction inside the separatrix (the separatrix is the curve that intersects the points marked by $\times$, which separate the closed and open field lines). The closed field lines is the region of containment. Outside the separatrix the field lines are open, and the plasma that reaches these outer lines, as well as plasma reaching the $z$-axis, flow towards the walls. By in large, the fux surfaces are produced by the toroidal plasma current. However, the hoop force still needs to be compensated for by an external vertical magnetic field, just as in the large aspect ratio case.

As an example, a prototype analytic solution for the flux $\psi_{p}$ without toroidal field, which satisfies Eq. (5) is

$$
\psi_{p}=4 \psi_{\max } \frac{R^{2}}{R_{0}^{4}}\left[R_{0}^{2}-R^{2}-\alpha^{2} z^{2}\right]
$$

where

$$
\frac{\psi_{\max }}{2 \pi}=\frac{R_{0}^{2}}{4}\left(\frac{\mu_{0} p_{0}}{2\left(1+\alpha^{2} / 4\right)}\right)^{1 / 2}, p=p_{0} \psi_{p} / \psi_{\max }
$$

$R$ and $z$ are radial axial cylindrical coordinates indicated in Fig. 6 and $R_{0}$ a constant. The plasma pressure is confined to the region $\psi_{p}>0$, and the curve $u_{p}=0$ defines the separatrix. For this solution the separatrix 
forms an ellipsoid of revolution with major axis $R_{0}$ in the radial direction, and $R_{0} / \alpha$ in the $z$-direction. The required external magnetic field, $\mathbf{B}_{\text {ext }}$ is directed in the $z$-direction. The needed external field, $B_{\text {ext }}$, can be expressed in terms of a beta condition $\beta_{\theta}=2 \mu_{0} p_{0} / B_{\text {ext }}^{2}$, with $p_{0}$ the pressure at the 0-point of Fig. 6 where the poloidal magnetic field vanishes. For a sphericallyshaped separatrix $(\alpha=1), \beta_{0}=\frac{45}{16}$, while for an axially elongated separatrix $(\alpha \rightarrow 0), \beta_{\theta}=1$.

Another type of compact toroid, known as the spheromak, is one where the plasma also carries poloidal current, so that toroidal magnetic field exists inside the containment region, but not outside the separatrix. In these configurations the role of kinetic pressure and magnetic field pressure from the toroidal field are nearly interchangeable. Now, both the kinetic pressure and toroidal magnetic field pressure maximize at the 0 -point and vanish at the separatrix.

A third type of compact toroid is astron [Christofolis, 1962]. This is a configuration where current carriers are energetic particles. In this case the external magnetic field, $B_{\text {ext }}$, confines the energetic particles in orbits that are essentially the betatron orbits that appear in the theory of particle accelerators. The dominant momentum flux associated with the orbits of this configuration is

$$
\mathbf{P}=m_{b}\left\langle n_{b} v_{\theta}^{2}\right\rangle \hat{\boldsymbol{\theta}} \hat{\theta}
$$

with $m_{b}$ and $n_{b}$ and mass and density of the energetic particles and $v_{\theta}$ the 
directed $\theta$-directed relocity component. Now Eq. (5) needs to be solved using Eq. (15) for $\mathbb{P}$. If the current formed by this beam of energetic particles is intense enough, the shape of the poloidal magnetic fields is similar to that obtained with the other compact toroid solutions.

\subsubsection{Inductive voltage drive}

The diverse toroidal concepts discussed thus far will only last a magnetic diffusion time unless magnetic flux is "pumped" into the configuration. The diffusive nature of the discharge can be inferred from combining Faraday's induction law, Ohm's law, and Ampére's law, which leads to a diffusion-like equation for the flux, $\psi_{p}$, which is essentially of the form,

$$
\frac{\partial \psi_{p}}{\partial t}=\frac{1}{\sigma \mu_{0}} \Delta \psi_{p}
$$

with $\sigma$ the electrical conductivity. Unless a source of flux is present, Eq. (16) predicts that the magnetic configuration decays with a characteristic time $\tau_{. M}$ given by $\tau_{. M} \approx \frac{\mu_{0} \sigma a^{2}}{4} \approx 4 \times 10^{-4} a^{2} T^{3 / 2}(\mathrm{eV}) / Z_{\text {eff }}$ sec where $a$ is the minor radius in meters, $T_{e}(\mathrm{eV})$ the electron temperature in electron volts and the electrical conductivity is $\sigma=1.3 \times 10^{3} T_{e}^{3 / 2}(\mathrm{eV}) / Z_{\text {eff }}$ siemens $/ \mathrm{m}$ with $Z_{\text {eff }}$ the mean atomic number of the discharge. Sample diffusion times are: $\tau_{M}=$ $6 \times 10^{-3} \mathrm{sec}$ for $a=0.2 \mathrm{~m}, \Gamma_{e}=50 \mathrm{eV}$ which are typical of startup parameters; $T_{M}=1.0 \mathrm{~min}$ for $a=1.0 \mathrm{~m}, T_{e}=3 \mathrm{keV}$ which are typical parameters for present-day tokamaks. $T_{H}=0.8 \mathrm{hrs}$. for $a=2 \mathrm{~m}, T=15 \mathrm{keV}$ which are typical of fusion reactor parameters. We observe that stronger magnetic fux 
pumping is required for small devices at low temperatures than for large devices at high temperatures.

The most obvious flux pump mechanism is to link magnetic flux, just as one does in a transformer, as shown in Fig. 7. The flux $\psi_{F}$ in the solenoid core is $\psi_{F}=B_{F} A_{F}$ where $B_{F}$ is the magnetic field in the solenoid and $A_{F}$ the cross-sectional area of the solenoid. If $B_{F}$ changes from $B_{F M}$ to $-B_{F M}$, it follows from Faraday's law that a voltage $V_{F}$ is imposed on the core plasma given by $V_{F}=\frac{-\partial \psi_{F}}{\partial t}=\frac{-\partial B_{F}}{\partial t} A_{F}$. In turn, the voltage is ohmically dissipated by the plasma, so that $V_{F}=-A_{F} \frac{\partial B_{F}}{\partial t}=I_{p} \mathcal{R}$ with $I_{p}$ the plasma current and $\mathcal{R}$ the plasma resistance $\left(\mathcal{R} \approx \frac{2 R}{\sigma a^{2}}\right)$. Because the total flux that can be pumped is limited to $2 A_{F} B_{F}$, then for a fixed current and resistance the solenoid can supply voltage for a time, $t_{F}$, given by $t_{F}=2 B_{F M} A_{F} / I_{p} \mathcal{R}$. This ohmic method of magnetic flux pumping will allow a reactor to operate on the scale of hours. Though quite long by today's standards, ohmic magnetic fux pumping implies periodic shut-down and start-up of the reactor, which is not desirable from several engineering considerations (one of the more significant reasons is that the many start-up phases lead to large changes in the wall temperature which cause continual thermal stress that eventually cause wall fracture). Hence, alternate flux pumping techniques, frequently called current drive, are desirable [Fisch, 1987]. For example, radio frequency waves have been used to drive steady plasma currents through a variety of mechanisms. Though less efficient than ohmic drive, they do offer the 
possibility of achieving truly steady state operation in a toroidal machine where plasma current is needed to form nested flux surfaces.

\subsubsection{Stellarators}

A way to avoid the need for driving current in a toroidal plasma is to design a stellarator configuration where flux surfaces form solely from the current in the external coils. The demonstration that "vacuum magnetic fields" form flux surfaces can be inferred from the properties of a periodic stellarator with helical symmetry and arbitrarily large aspect ratio. Consider a cylindrical coordinate system $(r, \theta, z)$, with the system extended in $z$, and define a phase variable $\psi=\theta-\alpha z$ with $\alpha$ a constant. The vacuum magnetic field can be expressed as $\mathbf{B}=\nabla \Phi$, which with $\nabla \cdot \mathbf{B}=0$, requires Laplace's equation to be satisfied, $\Delta \Phi=0$. A helical stellarator solution has the form

$$
\Phi=B_{0} z+\frac{1}{\alpha} \sum_{n=1}^{\infty} b_{\ell} I_{\ell}(\ell \alpha r) \sin (\ell \psi)
$$

where $I_{p}(x)$ is the Bessel function of imaginary argument. The field lines generated by these solutions lie tangent to a helical flux function $\psi_{H}$,

$$
\psi_{H}=B_{0} \frac{\alpha r^{2}}{2}-r \sum_{\ell=1}^{\infty} b_{\ell} I_{\ell}^{\prime}(\ell \alpha r) \cos \ell \theta \text {. }
$$

These solutions are the first approximations to the fields that can be generated in a torus with a large aspect ratio $\left(R_{0} / a \gg 1\right.$ where $R_{0}$ and $a$ are typical major and minor radii). Helical solutions are obtained by winding the external current carrier in a helix $. M / \ell$ times around poloidally for each toroidal 
turn, with $M$ an integer and $\alpha=M / \ell R_{0}$. The pitch of the coil is $M / \ell$. Examples of the shapes of constant helical flux contours at fixed $z$ generated from various coil configurations, are given in Fig. 8. At a given poloidal cross-section there are $\ell$-current paths flowing in one direction (shown by the symbol $\oplus$ in Fig. 8a) and perhaps $\ell$-current paths in the opposite direction (shown by the symbol $\theta$ ). If the latter set of currents are absent the configuration is called a heliotron or torsatron; with a torsatron a single coil forms toroidal and helical fields. The magnetic field lines inside the torus wind around the magnetic axis with a rotational transform that is less than the pitch of the current loops. The rotational transform increases with increasing radial distance until a separatrix is reached (in these figures the separatrix are the contours that cross on themselves), where the rotational transform equals the pitch. At radii outside the separatrix, field lines are not contained, but intersect the wall. Unlike a typical tokamak, $q=1 / \iota$ decreases away from the magnetic axis.

In practice a straight system must be wrapped into a large aspect ratio torus as in Fig. 8b. Observe that the magnetic flux surfaces twist to follow the external helical currents. However, strict helical symmetry is broken by the toroidal shape. With strict helical symmetry it follows from Eq. (18) that the magnetic fields inside the separatrix have nested flux surfaces. With helical symm " $y$ broken (as it must be in a true toroidal symmetry), it is not at all obvious that a field line generates a magnetic flux surface rather than filling a 
volume of space. Fortunately, the flux surface property is retained over a large volume surrounding the magnetic axis. However, flux surfaces disappear near the separatrix (there field lines meander over a finite volume) and additional structure in the field lines (so-called magnetic islands) arise near surfaces with rational rotational transform. With proper coils, an acceptable volume of mostly nested magnetic surfaces can be designed. Within these good surfaces, plasmas can be retained to beta values comparable to what is possible with tokamaks.

Historically, the stellarator concept was one of the pioneering confinement concepts in controlled fusion. The three-dimensional aspect of the configuration made design and analysis difficult, and progress in the field (in part due to poor design choices) lagged behind tokamak research. In more recent years, as a result of clearer conceptual ideas of the stellarator concept, very promising experimental results have emerged in stellarator research. Today, the stellarator concept, with its multiple design options and its intrinsic truly steady state nature, is a leading alternate to tokamaks as a means for achieving fusion-grade plasmas [Carreras, et al., 1988]. 


\section{PARTICLE ORBITS AND CLASSICAL TRANSPORT IN MAG- NETICALLY CONFINED PLASMAS}

\subsection{Particle Orbits in Magnetic and Electric Fields}

Without magnetic and electric fields or collisions, a particle in an enclosed volume moves in a straight line until it hits the surrounding wall. Magnetic and electric fields can be designed to prevent the particles from hitting bounding surfaces. The orbits are determined from the equations of motion,

$$
\mathbf{v}=\frac{d \mathbf{r}}{d t} ; \quad M_{j} \frac{d \mathbf{v}}{d t}=e_{j}(\mathbf{v} \times \mathbf{B}+\mathbf{E})
$$

with $M_{j}$ and $\epsilon_{j}^{\prime}$ the mass and charge respectively of species $j$. When $\mathbf{B}$ is spatially uniform and $\mathbf{E}=0$, the particle motion perpendicular to the magnetic field is a circle with a radius $\rho_{j}=v_{\perp} / \omega_{c j}$, (known as the Larmor radius) about a point called the guiding center, $v_{\perp}$ the speed of the velocity component perpendicular to the magnetic field and $\omega_{c}=e_{j} B / M_{j}$ is the cyclotron frequency of species $j$. However, the parallel velocity, $v_{\|} \equiv \mathbf{v} \cdot \mathbf{B} / B$, remains constant and the particle moves without containment parallel to the magnetic field in a manner shown in Fig. 9a. With a homogeneous static electric field present perpendicular to the magnetic field, a particle takes on an additional drift motion, $\mathbf{v}_{E}=\mathbf{E} \times \mathbf{B} /|B|^{2}$, giving rise to a motion shown in Fig. 9b, with the motion of the guiding center being perpendicular to the direction of $\mathbf{E}$.

The motion we have described has not taken into account collisions be- 
tween particles due to the binary Coulomb interaction. Because of the longrange nature of the Coulomb potential, the many small angle deflections arising from collisions with relatively large impact parameters, are more important than the large-angle-deflections arising from collisions with small impact parameters. The small angle deflections cause the particle pitch angle $\theta=\cos ^{-1} v_{\|} /|\mathbf{v}|$ of a species, $j$, to change by $90^{\circ}$ in a characteristic scattering time $\tau_{j}$, which is given by

$$
\begin{aligned}
& n_{e} T_{i} \approx 1.5 \times 10^{10} \mathrm{~A}^{1 / 2} E_{i}(\mathrm{keV}) / Z^{3} \\
& n_{e} T_{e}=1.5 \times 10^{10}\left(M_{e} / M_{i}\right)^{1 / 2} E_{e}^{1 / 2}(\mathrm{keV}) /(1+1.5 Z)
\end{aligned}
$$

where $E_{j}(\mathrm{keV})$ is the energy of species $j$ in $\mathrm{keV}, j \equiv i$ refers to ions, $j \equiv e$ refers to electrons, $A$ and $Z$ refer to the ions' atomic mass and number respectively, and we assume only one ion species for simplicity.

As a particle scatters, the guiding center of a particle likewise deflects, and in a scattering time $t=\tau_{j}$, the guiding center is deflected by roughly one Larmor radius. For time scales much longer than $\tau_{j}$ the evolution across field lines is described by random walk theory. The diffusion coefficient, $D_{j}$, for species $j$ is given by $D_{j}=\Delta x_{j}^{2} / \Delta t$ with $\Delta x_{j}$ is the random step size that occurs after a time $\Delta t$. In our case, $\Delta x=\rho_{j}$ and $\Delta t=\tau_{j}$, which gives the "classical" diffusion coefficient of a particle in a straight magnetic field,

$$
D_{j} \approx \frac{\rho_{j}^{2}}{\tau_{j}} .
$$

When collisions are taken into account in fields where $\mathbf{E}$ and $\mathbf{B}$ are mutually perpendicular, particle diffusion across field lines gives rise to a thermal 
conduction coefficient $\chi \sim D_{i}$. As a result, for a slab model of a plasma, with the magnetic field in the $z$-direction and an electric field in the $x$ direction, and with the plasma confined in $x$ within a characteristic spatial scale $L_{p} \gg \bar{\rho}_{i}\left(\bar{\rho}_{i}=\sqrt{2 T_{i} / m_{i}}\right)$, the characteristic lifetime for energy confinement, $\tau_{\text {th }}$ is

$$
\tau_{\mathrm{th}} \approx \frac{L_{p}^{2}}{\gamma_{i}} \approx \frac{L_{p}^{2} \bar{\tau}_{i}}{\bar{\rho}_{i}^{2}}
$$

where for $\bar{\tau}_{i}$ we use Eq. (20) with $E_{i}=T_{i}$. As $L_{p} \gg \bar{\rho}_{i}$, the energy lifetime is many $90^{\circ}$ deflection times.

In addition collisions prevent the unbounded acceleration of particles when a parallel electric field is present. With collisions the parallel equation of motion gives roughly

$$
\frac{d v_{\|}}{d t}=e_{j} \frac{E_{\|}}{M_{j}}-v_{\|} / \tau_{j}
$$

with a steady-state solution $v_{\|}=e_{j} E_{\|} \tau_{j} / M_{j}$ which produces a plasma current

$$
j_{\|}=-e n_{e}\left(v_{\| e}-v_{\| i}\right) \approx \tau_{e} n_{e} e^{2} E_{\|} / M_{j} \equiv \sigma E_{\|}
$$

with $e$ the magnitude of the electron charge. Within a numerical coefficient this value of $\sigma$ reproduces the electrical conductivity used in Eq. (16).

\subsection{Spatially Varying Fields}

By considering magnetic and electric fields that vary slowly in space compared to a Larmor radius, and slowly in time compared to a cyclotron period 
$\sim 1 / \omega_{c}$, one can demonstrate particle containment along the magnetic field. Under these conditions the quantity $i \because \equiv m\left(\mathbf{v}_{\perp}-\mathbf{v}_{E}\right)^{2} / B$ is an adiabatic invariant, i.e. it remains conserved to high accuracy during a particle's motion. As a consequence of this invariance, the parallel velocity of the guiding center, $v_{\|}$, is given by

$$
v_{\|}= \pm \sqrt{\frac{2}{M_{j}}}\left(\mathcal{E}-\mu B(s)-e_{j} \Phi(s)\right)^{1 / 2}
$$

where $-\nabla \Phi=\mathbf{E}, \mathcal{E}=\frac{1}{2} m v^{2}+\epsilon_{j} \Phi$ is the total conserved particle energy, $B(s)=|\mathbf{B}|$ and $\Phi$ is the electrostatic potential. From Eq. (23) it is clear that if the magnetic field is increasing, a particle can be contained along the field line. Specifically, when $\Phi=0$, the particle containment condition is

$$
\frac{v_{\perp 0}^{2}}{v^{2}}>\frac{B_{0}}{B_{\max }} \equiv \frac{1}{R}
$$

where $B_{\max }$ and $B_{0}$ are respectively the maximum and mid-plane values of the magnitude of the magnetic field, $v_{\perp 0}$ is the speed of the velocity component perpendicular to the magnetic field, the subscript 0 refers to its value at the mid-plane, and $R$ is called the mirror ratio. This type of containment is referred to as mirror containment, where particles that satisfy Eq. (24) oscillate between two turning points inside the mirror peaks (for an axially symmetric machine where $B(-s)=B(s)$ the turning points are at $s= \pm s_{T}$ where $\left.B\left(s_{T}\right) / B_{0}=v^{2} / v_{\perp 0}^{2}\right)$. Particles for which $v_{\perp 0}^{2} / v^{2}<1 / R$ are in the so-called loss cone and they leave the mirror machine in one transit time.

The particles that satisfy Eq. (24) are axially trapped and remain there 
for many axial bounce periods. The limiting lifetime, $\tau_{s c}$, is determined by collisions causing a particle to pitch-angle scatter into the loss cone. This time is roughly $\tau_{s c} \sim \tau_{j}(1-1 / R)$ for $R-1 \ll 1$ and $\tau_{s c} \sim r_{j} \ln R$ for $R \gg 1$, with $\tau_{j}$ given by Eq. (20).

In the absence of parallel electric fields, electrons are lost more rapidly than ions as $\tau_{s c e} / \tau_{s c i} \approx\left(M_{e} / M_{i}\right)^{1 / 2}\left(T_{e} / E_{i}\right)^{3 / 2}$. Hence the plasma will charge up positively, producing an axial electric field pointing outward towards the mirror peaks. This field reinforces the containment of electrons and weakens the containment of ions. The magnitude of the negative potential adjusts so that the axial losses of electrons and ions will be equal. Thus the electrostatic potential is of intrinsic importance in the containment of plasma in a mirror machine. It is referred as an ambipolar potential as it regulates the charged particle loss to occur with nearly zero current flow.

The containment condition for ions and electrons with an ambipolar potential is obtained using Eq. (23) by demanding that the turnirig point $s_{T}$ lie inside the mirror peak position at $s=s_{\max }$. This modifies the containment condition given by Eq. (24), to,

$$
v_{\perp 0}^{2}>\frac{v_{0}^{2}}{R}+\frac{2 e_{j} \Delta \Phi}{M_{j} R}
$$

where $\Delta \Phi=\Phi(0)-\Phi\left(s_{\max }\right)$. For ions, where the charge $e_{j}=Z e$ is positive, we see that all ions with kinetic energy in the mid-plane less than $e_{j} \Delta \Phi /(R-$ 1) $\equiv E_{\text {crit }}$ are lost in one transit.

On the other hand, for electrons, where $e_{j}=-\epsilon$ is negative, the con- 
tainment region is enhanced. All low energy particles for which $\frac{1}{2} M_{\mathrm{e}} v_{0}^{2}<$ $e\lrcorner \Phi /(R-1)=E_{\text {crit }}$ are trapped independent of pitch angle. Such particles can only be lost if during their diffusion process they gain energy. Electrons with mid-plane kinetic energies greater than $E_{\text {crit }}$ are lost by pitch-angle scattering caused by collisions.

In mirror machine operation the ambipolar-potential $e \Delta \Phi$ is generally several times the electron temperature $T_{e}$, which, as we shall see, is an order: of magnitude less than the ion mean thermal energy. Hence, most electrons are trapped independent of pitch angle, where they remain for many collision times. Thus the bulk of electrons relax to nearly a Maxwell distribution. Only a high energy tail of electrons whose density is roughly $n_{e} \exp \left(-e \Delta \Phi / T_{e}\right)$, do not thermalize since these electrons are lost by pitchangle scattering. Hence, the effective rate of loss, $\nu_{e}(\Delta \Phi)$, of electrons with an ambipolar potential present is roughly

$$
\nu_{e}(\Delta \Phi) \approx \frac{1}{T_{e}} \exp \left(-\frac{e \Delta \Phi}{T_{e}}\right) .
$$

If $q \Delta \Phi /(R-1) \lesssim E_{i}$, the ion loss rate is somewhat reduced, but basically comparable to $1 / \tau_{i}$ given by Eq. (20). Thus equating the electron and ion loss rates, we find that the ambipolar potential is roughly given by

$$
\frac{\Delta \Phi}{k \cdot T_{e}} \approx \ln \left(\left(\frac{M_{i}}{M_{e}}\right)^{1 / 2}\left(\frac{E_{i}}{T_{e}}\right)^{3 / 2}\right)
$$

Typically this factor $\sim j$ while $E_{i} / T_{e} \sim 10$. The reason why such a large ratio of $\bar{E}_{1} / T_{e}$ can be maintained in steady state is because a typical ion is 
lost with an energy somewhat less than its mean value $\bar{E}_{i}$, while a typical electron is lost with a mean energy $e \boldsymbol{\Phi}+T_{e} \gg T_{e}$.

\subsubsection{Q-value in mirror machine}

The ratio of the power produced by fusion to the power lost by classical collisions is frequently referred to as $Q$. Detailed solutions of the Fokker-Planck equation (the equation that describes the diffusive evolution of particle distribution functions due to multiple small angle scattering) in mirror geometry show that $Q$ can only slightly exceed unity for the $\mathrm{d}$ - $t$ reaction. This result severely limits using a simple mirror machine for net energy production from the fusion reaction. Therefore, various methods to enhance the efficiency of energy production have been proposed. One simple procedure is to directly convert the energy flux of outgoing ions to electrical potential energy so that the effective $Q$ value then becomes $Q_{0} /(1-\eta)$ where $Q_{0}$ is the original $Q$ value and $\eta$ is the ultimate fraction of the outgoing energy that can be reintroduced into the plasma ( $\eta \sim 0.7$ is a realistic expectation).

\subsubsection{Electrostatic potentials in tandem mirrors}

An alternative idea is the tandem mirror concept, where parallel electric fields are actively used to enhance the containment. A schematic of a basic tandem mirror configuration is shown in Fig. 10. There is a central cell mirror containing the bulk of the energy where the potential is negative with respect to the surrounding end cells so that ions can be contained, while the end cells 
are separate mirror machines whose electrostatic potential with respect to the wall is positive so that electrons are contained as they are in a conventional mirror machine. This potential structure can be established if the mean ion energy in the end plugs is larger than the central cell ion temperature. However, because of the much larger volume of the central cell, most of the energy is stored there. The role of end plugs is to serve as a containment dam that comes from the electric field structure. Pitch-angle scattering of ions in the central cell does not cause particle ion loss, while the potential between the plasma and the end wall prevents pitch-angle scatteriı, of electrons from causing particle loss. The bulk losses only arise from pitch angle scattering of ions and electrons in the high energy tail. The ions in the plugs are susceptible to the usual mirror losses, but their low inventory of total stored energy diminishes the seriousness of these losses. As a result the $Q$ value of a tandem mirror can be increased substantially above that predicted for a simple mirror machine. Even further improvement in $Q$ can be designed by actively controlling the ambipolar potential and forming so-called thermal barriers. The dotted curve in Fig 10 indicates how the potential would look like with a thermal barrier. With a thermal barrier present, the temperatures of electrons in the plug and central cell can be decoupled, which ultimately allows the ions in the plug to have a mean energy comparable to that in the central cell. Hence the parasitic losses due to collisions in the plug is not as severe as in the more conventional tandem mirror, and the $Q$-value can be 
improved still further.

\subsection{Orbits in Toroidal Field}

The alternative to an open ended system is to have a toroidally shaped plasma. Then to the extent particles follow field lines, one would expect containment inside the torus. However, toroidal containment is more subtle as particle trajectories differ somewhat from the trajectory of a magnetic field even in the absence of electric fields. For example, consider the simplest magnetic field to return on itself, which arises from a straight wire, and has the form

$$
\mathbf{B}=B_{\phi} \frac{R_{0}}{R} \hat{\phi}
$$

where $\phi$ is the toroidal angle about the $z$-axis, $\hat{\phi}$ a unit vector in the $\phi$ direction, $R$ the major radius, and $R_{0}$ a convenient constant denoting the typical major radius. A magnetic field line in such a field is a circle of radius $R$. However, such a field does not allow charged particles to be contained in a closed volume. The magnetic field given by $\mathrm{Eq}$. (27) has a finite magnetic field gradient $\frac{1}{|B|} \boldsymbol{\nabla}|B|=-1 / R \widehat{\mathbf{R}}$ and field line curvature $(\mathbf{b} \cdot \boldsymbol{\nabla}) \mathbf{b}=-\widehat{R} / R$ where $\mathbf{b}=\mathbf{B} /|B|$ is the unit vector along a magnetic field line (note that in vacuum magnetic fields where $\boldsymbol{\nabla} \times \mathbf{B}=0, \mathbf{b} \times(\mathbf{b} \cdot \boldsymbol{\nabla}) \mathbf{b}=\mathbf{b} \times \boldsymbol{\nabla}|B| /|B|)$. From Eq. (19) one can show that magnetic gradients and field line curvature produce an orbit drift velocity, $\mathbf{v}_{D}$, transverse to the magnetic field given by

$$
v_{D}=\frac{v_{\| l}^{2}}{\omega_{c}} \mathbf{b} \times(\mathbf{b} \cdot \nabla) \mathbf{b}+\frac{v_{1}^{2}}{2 \omega_{i}} \frac{\mathbf{b} \times \nabla|B|}{B} .
$$


In the field given by Eq. (28), particle trajectories primarily follow the $\hat{\phi}$ directed field, but in addition the drift velocity in Eq. (28) causes the orbits to drift indefinitely in the negative $\hat{z}$-direction transverse to the magnetic field with a speed

$$
\mathrm{v}_{D}=\left(\frac{v_{\perp}^{2}}{2}+v_{\|}^{2}\right) / \omega_{c} R .
$$

To prevent unbounded motion a poloidal magnetic field needs to be present. One simple way of establishing such a field is with a current ring carrying a current $I_{\phi 0}$ at a major radius $R=R_{0}$, which gives a magnetic field $\mathbf{B}_{\theta}=B_{\theta 0} \hat{\boldsymbol{\theta}} r_{0} / r$ if $r \ll R$, with $B_{\theta 0}=\mu_{0} I_{\phi_{0}} / 2 \pi r_{0}$, where $r$ is the minor radius and $r_{0}$ is a constant. Such a configuration is referred to as a hard core pinch or levitron. Alternatively, the toroidal current can be produced by the various toroidal diffuse pinches where the plasma carries toroidal current, as described in the previous section. The magnetic field then has the generic form

$$
\mathbf{B}=B_{\phi} \frac{R_{0}}{R}(\hat{\phi}+q(r) \hat{\boldsymbol{\theta}})
$$

with $q(r)$ the inverse rotational transform. In Fig. 11 we show typical drift orbits of positive charged particles in this field projected unto a $\phi=$ constant plane when $v_{\| l} / v \gg(r / R)^{1 / 2}$. In addition, not shown in the figure, there is rapid oscillatory motion about the drift trajectory due to the Larmor motion. Figure $11 \mathrm{a}$ is for passing particles, a terminology that applies to those particles where $v_{\|} / v>(2 r / R)^{1 / 2}$. These particles always circulate in a fixed $\theta$-direction. The dotted line denotes the magnetic flux surfaces 
formed from the magnetic field given by $\mathrm{Eq}$. (30). One should note that co-moving particles i.e. those particles whose sense of motion produces a current along the magnetic field that is in the same sense as the prevailing current (in Fig. 1la the prevailing current points towards the reader) is shifted inwards, while counter-moving particles (i.e.those particles that move in the opposite direction) are shifted outwards. This asymmetry produces a bias that helps sustain current in a toroidal discharge. This current bias also exists in the electron motion. The trajectory's structure can be understood by noting that the particles' velocity is primarily tangential to the flux surface. However, the additional drift velocity causes a positively charged particle moving in the clockwise direction to move radially away from the flux surface when it is below the mid-plane. This outward displacement is compensated when the particle is above the mid-plane where the drift velocity takes the particle back to the flux surface. A similar argument holds for the counterclockwise rotating particle. The overall excursion from the flux surface $\delta r \approx$ $q(r) \rho(r) \frac{\left(v_{\|}^{2}+v_{\perp}^{2} / 2\right)}{v_{\|} v_{\perp}}$ (recall $\rho$ is the Larmor radius $v_{\perp} / \omega_{c}$ ) is inward for "comoving" particles (particles moving parallel to the current) and outward for "counter-moving" particles as indicated in Fig. 11 a.

On a flux surface the magnetic field varies, and the mirror ratio on the flux surface is approximately

$$
R=\frac{B_{\phi}\left(R=R_{0}-r\right)}{B_{\phi}(R=R+r)}=\frac{1+\epsilon}{1-\epsilon} \approx 1+2 \epsilon
$$

with $\epsilon=r / R$ the inverse aspect ratio. If $v_{\|}^{2} / v_{\perp}^{2}<2 \epsilon$, particles are mirror 
trapped on the flux surface and they are constrained to remain on the outside of the torus. They are referred to as "trapped" particles. The orbit of a trapped particle, projected unto a cross-sectional plane where $\phi$ is constant, is shown in Fig. 11b. Because of the shape of the trajectory, the orbit of a trapped particle is referred to as a "banana" orbit. These orbits are still contained in a finite volume, but their orbit width given by

$$
\delta r \approx \frac{q \rho}{\epsilon^{1 / 2}} \equiv r_{b}
$$

is somewhat larger than a passing particle.

\subsection{Neoclassical Diffusion}

The complications of the orbit structure in a toroidal magnetic field cause an enhancement of the previously estimated classical diffusion. The appropriate theory is called neoclassical diffusion [Galeev, et al., 1979] and we now present a heuristic discussion of its implications.

Using random walk theory, we estimate the diffusion coefficient of a particle by noting that a particle will diffuse an orbit width of its trajectory, $\Delta r$, in an effective collision time $\tau_{\text {eff }}$. The diffusion coefficient, $D$, can be estimated as,

$$
D=\Delta r^{2} / \tau_{\text {eff }}
$$

while the overall energy containment time is then given by

$$
\tau_{E} \approx \frac{a^{2}}{D}=\frac{a^{2}}{\Delta r^{2}} \tau_{\mathrm{eff}}
$$


The majority of particles are passing and their effective relaxation time is a $90^{\circ}$ scattering time $\tau_{j}\left(\tau_{j}\right.$ is either $\tau_{i}$ or $\tau_{e}$, given in $\mathrm{Eq} .(20)$, depending on the type of particle), while their mean displacement is $\Delta r \approx q \rho$. Hence, the diffusion coefficient for passing particles, $D_{\text {pass }}$, is given by $D_{\text {pass }} \approx q^{2} \rho_{j}^{2} / \tau_{j}$, with $\rho_{j}$ the Larmor radius of species $j$. However, this value for the diffusion coefficient turns out to be too small. Surprisingly, it is the diffusion of the trapped particle minority that primarily determines the containment characteristics. This is because $\Delta r$ is larger for a trapped particle than for a passing particle and $\tau_{\text {eff }}$ is shorter. For a trapped particle $\Delta r \approx q \rho / \epsilon^{1 / 2}$, which is the characteristic deviation of a trapped particle from a flux tube, and $\tau_{\text {eff }} \approx \tau_{j} \epsilon$, which is the characteristic time a trapped particle remains trapped. The shorter $\tau_{\text {eff }}$ arises because of the diffusive nature of the Coulomb collisional process. Hence if $\tau$, is the time for a particle to diffuse $90^{\circ}$ in velocity space, $\tau_{j} \sin ^{2} \Delta \theta$ would be the characteristic time to diffuse a pitch angle width $\Delta \theta$; for trapped particles $\sin \Delta \theta \approx R-1 \sim \epsilon^{1 / 2}$ where $R$ is the mirror ratio on the flux tube of a tokamak. Hence the diffusion coefficient of a trapped particle is $D_{\text {trap }} \simeq \Delta r^{2} / \tau_{\text {eff }} \simeq q^{2} \rho^{2} / \epsilon^{2} \tau_{j}$. The fraction of phase space that is occupied by trapped particles is approximately $\sin \Delta \theta \simeq \epsilon^{1 / 2}$, and hence the global diffusion coefficient due to both trapped and passing particles is:

$$
D \approx \epsilon^{1 / 2} D_{\text {trap }}+D_{\text {pass }} \approx \frac{q^{2} \rho^{2}}{\tau_{j}}\left(\frac{1}{\epsilon^{3 / 2}}+1\right) \text {. }
$$

Clearly the contribution to the $D$ from the trapped particles is more important than from the passing particles. The global energy lifetime due to 
thermal conduction is then determined by

$$
n \tau_{E} \approx \frac{a^{2} n}{D} \approx \frac{a^{2}}{\rho_{i}^{2} q^{2}} \frac{n \tau_{i}}{\left(a / R_{0}\right)^{3 / 2}}
$$

with $\rho_{i}$ the ion Larmor radius. The large factor $a^{2} / \rho_{i}^{2}$ ensures that the intrinsic thermal conduction losses in a tokamak can satisfy the Lawson criterion for ignition by a large margin.

\subsubsection{Bootstrap current}

The so-called bootstrap current is another important collisional effect. It arises because: (1) co-rotating particles tend to be shifted inward while counter rotating particles are shifted outward; (2) the primary diffusion process is significant only when particles are trapped or nearly trapped. As a consequence the statistical weight of a particle, even when it is passing, is determined by its weight when it was trapped. At a given radius $r$ the co-circulating passing particles then have a weight of trapped particles that were centered around an inward shifted radius $r-q \rho / \epsilon^{1 / 2}$, while a counter-circulating particle has an equal weight shifted to the outward radius $r+q \rho / \epsilon^{1 / 2}$. The current density at the flux surface $r$ is then given by

$$
j_{\|}=e_{j}\left(n^{+}(r)-n^{-}(r)\right) v_{t h}
$$

where $n^{+}$is the density (proportional to the statistical weight) of co-moving particles and $n^{-}$the density of counter moving particles. Using $n^{+}(r+$ 
$\left.2 q \rho / \epsilon^{1 / 2}\right) \approx n^{-}(r)$ we find the bootstrap current density

$$
j_{\|} \approx-2 e, \frac{\partial n}{\partial r} \frac{q \rho}{\epsilon^{1 / 2}} v_{t h}
$$

This can be a substantial fraction of the current required to maintain a discharge. It allows for a substantial decrease in the current drive needed to maintain a steady-state tokamak.

\subsubsection{Ware pinch}

As the bootstrap current results from a spatial gradient that "drives" current, it follows from an Onsager relation that a toroidal electric field will induce a radial spatial gradient. The phenomena is called the Ware Pinch.

In this phenomenon the $\widehat{\phi}$-directed electric field that is being used to drive current is not quite parallel to the magnetic field. For passing particles there is an inward flow speed $\mathbf{E} \times \mathbf{B} /|B|^{2} \approx E_{\phi} B_{\theta} /|B|^{2}$. However, the flow speed is too slow for trapped particles, which actually move inward at the speed $E_{\phi} / B_{\theta}$. This enhanced speed arises for the following physical reason.

If we use Eq. (23) to determine the turning of a trapped particle with the electric potential given by $\Phi=\int^{s} d s^{\prime} E\left(s^{\prime}\right)$, one finds that the turning points are displaced. As a result, a banana orbit is shifted as shown in Fig. 11c. In the figure shown, the particle spends more time above the mid-plane than below the mid-plane. The downward curvature drift then directs the particle towards the flux surface more frequently than away from the flux surface. As a result after averaging over a particle's bounce orbit, a trapped particle will 
move inward at an inward radial speed given by

$$
\dot{r}=-\frac{E_{\phi}}{B_{\theta}}
$$

where $E_{\phi}$ is taken positive if it is in the direction of the prevailing toroidal current. As only trapped particles with a density $n_{T} \approx(r / R)^{1 / 2} n_{i}$ move at this rate, the actual inward flux is

$$
n_{T} \bar{\nu}_{r} \approx n_{i}\left(\frac{r}{R}\right)^{1 / 2} \frac{E_{\phi}}{B_{\theta}} .
$$

\subsection{Radial Electric Field}

The effect of radial electric field can also cause a profound change in particle containment. Because electrons move rapidly along the field lines forming flux surfaces, there is a strong tendency for a magnetic flux surface to be an electric potential surface. Consequently, the strongest electric fields in a tokamak tend to be normal to the flux surface. Such an electric field produces the standard $\mathbf{E} \times \mathbf{B}$ drift, whose flow is tangential to a flux tube, nearly in the $\theta$-direction. This motion alters the characteristic displacement of a particle from a flux surface. Without an electric field, the magnetic drift motion of particles causes a typical radial flow speed $v_{r} \sim\left(v_{\|}^{2}+\frac{v_{1}^{2}}{2}\right) / \omega_{r} R$ that alternates towards and away from the flux surface, with a radian frequency $\omega_{b} \approx v_{\|} / q R$ in the poloidal direction, giving rise to a displacement $\delta r_{b}=$ $v_{r} / \omega_{b} \approx q\left(v_{\|}^{2}+v_{\perp}^{2} / 2\right) / \omega_{c} v^{\prime} \|$ from the flux surface. With a radial electric field present, the particle has an additional poloidal velocity component $v_{E \theta} \approx$ 
$c E_{r} / B$, which should be added to the $\theta$-component of the parallel flow, giving a total $\theta$-component flow speed $v_{\theta}=\frac{\iota}{q}\left(v_{\|}+q v_{E \theta} / \epsilon\right)$. Hence the circulation frequency is $\omega_{b} \approx v_{\theta} / r=\left(v_{\|} \|+q v_{E \theta} / \epsilon\right) / q R$, and the radial displacement is $\delta r_{b} \approx q\left(v_{\|}^{2}+v_{\perp}^{2}\right) /\left[\omega_{c}\left(v_{\|}+q \frac{v_{E} \theta}{c}\right)\right]$. Without a radial electric field, the largest particle displacement occurs for small $v_{\|} / v$ where the distribution of particles peak. The radial electric field changes the region of phase space for which the displacement from a Hux tube is maximum. Instead of $v_{\|} \approx 0$, the displacement maximizes at $v_{\|} \simeq v_{E \theta} / \epsilon$. It is then possible for the large particle displacement to affect only particies in the tail of the distribution function where there are relatively few particles, while all the particles in the bulk remain close to the magnetic surface.

Recent tokamak experiments, e.g. [Asdex Team, 1989], have found that there is a profound improvement of plasma containment from more usual discharges in the so-called $H$-mode regime. In this regime the radial electric field in the plasma near the edge is found to satisfy the condition $v_{t h}<$ $q v_{E \theta} / \epsilon$, and the radial electric field appears to be an essential element in controlling particle transport.

\subsection{Orbits in Stellarators}

In stellarators, where toroidal symmetry is broken, particle containment can differ profoundly from systems with toroidal symmetry. In the latter case with a finite rotational transform, the particle displacement from a flux tube 
is guaranteed to be some factor of a Larmor radius. Hence with toroidal symmetry present, as $B$ becomes larger the particle displacement from the flux surface becomes smaller. The lack of toroidal symmetry allows special classes of particles to deviate substantially from the flux surfaces, to distances that can be comparable to the machine size itself, independent of the magnitude of the magnetic field. In the absence of electric fields, the particles most susceptible to this large deviation are those that are mirror trapped by the magnetic field modulations due to the combined toroidal and helical field structure. They form so-called super banana orbits, where in one bounce time the orbit appears like a trapped banana orbit. As the magnetic moment is conserved, the banana orbit moves with the magnetic field at the banana tip, remaining constant. This constraint in itself does not guarantee orbit containment. Thus, many stellarator configurations in the absence of radial electric fields have inherent lo:s regions. With a radial electric field present, orbits will remain closer to flux surfaces, and containment can be improved. However, as the electrostatic potential tends to be comparable to the temperature of the plasma, it is very difficult to modify the containment properties of the energetic particles. This particularly applies to the charged fusion products that need to be retained in a reactor during ignition. Hence the large orbit displacement from a flux surface will give rise to a direct particle loss of a relatively large fraction of the fusion products in a stellarator (perhaps $\sim 25 \%$ ). Such a factor does not quench ignition scenarios, but 
does introduce additional problems. For example, losses tend to be concentrated in specific places of the wall, which induces very special heat load and sputtering problems that need to be addressed in reactor designs.

\section{PLASMA INSTABILITY AND ANOMALOUS TRANSPORT}

A plasma is characterized by several intrinsic and disparate spatial and temporal scales. Hence, even in the same system, several different types of instabilities can arise that cause different responses. Long wavelength instability can cause rapid disintegration of a configuration, or can establish a threshold which prevents operation above a critical beta value. Short wavelength instability tends to saturate at relatively low perturbed field values, so that while the integrity of a system can still be maintained, the plasma transport is frequently enhanced above intrinsic losses.

\subsection{Ideal MHD Theory}

A first approach to describe plasma stability is to use ideal magnetohydrodynamic (MHD) theory. In this model, viscosity, resistivity and heat conduction are ignored, and the plasma pressure satisfies the ideal gas law. In Sec. 3 we discussed equilibria that arise from the MHD equations. If these equilibria are perturbed, the time-dependent response determines whether small perturbations remain small or grow to finite values. The former case implies stability, while the latter situation implies instability. 


\subsubsection{Simplified dynamical model}

When a plasma is described by the ideal MHD model, the method of determining stability is a generalization of the principle of virtual work, that is readily illustrated in a one-dimensional system with two degrees of freedom. A potential $V(\xi)$ is given, say, in the shape of Fig. 12 with $\xi$ the position and $V$ the potential. The equation of motion is given by

$$
\frac{d^{2} \xi}{d t^{2}}+\frac{\partial V(\xi)}{\partial \xi}=0
$$

In Fig. 12, equilibrium points exist at positions (a), (b), (c), and (d), where $\partial V / \partial \xi=0$.

From the principle of virtual work the stability of the equilibrium points are determined from the sign of the perturbed potential energy,

$$
\delta W \equiv \delta \xi^{2} \frac{\partial^{2} V\left(\xi_{i}\right)}{\partial \xi^{2}}
$$

where $\xi_{i}$ is the equilibrium point where $\frac{\partial V\left(\xi_{i}\right)}{\partial \xi}=0, \delta \xi \equiv \xi-\xi_{i}$ is an arbitrarily small displacernent, from $\xi_{1}$ and $\delta W$ is the virtual work that must be applied to displace the system. If $\delta W>0$ for every arbitrarily small displacement, the system is locally stable and an initially small perturbation will remain small, while if $\delta W<0$, an initially small perturbation will grow to finite and perhaps even infinite amplitude. For this one-dimensional system the sign of $\delta W$ is equivalent to determining the sign of $\partial^{2} V^{\prime} / \partial \xi^{2}$.

In the simple system shown in Fig. 12 it is clear that initially small deviations about points (a) and (c) lead to finite deviations from these points, 
while srnall deviations from points (b) and (d) remain small. The system illustrated here is simple enough that we can ascertain that a small deviation about point (c) leads to a bounded oscillation between points $\alpha$ and $\beta$. With a small amount of dissipation present, one expects a state initially at (c) to relax to either points (b) or (d). However a small deviation about point (a) can quickly lead to an unbounded excursion. Hence this simple example illustrates how instability can lead to rapid disruptions or to a nonlinear bounded state that can eventually relax to a stable state.

\subsubsection{Generalization to plasmas}

These relatively simple considerations can be applied to more complicated systems with many degrees of freedom and even to systems where the degrees of freedom form a continuum, as it does with the MHD equations; see for example [Friedberg, 1982] for a detailed derivation and detailed application of the method. If one can show that the virtual work (or as it is frequently called, the MHD energy) is positive for every possible small displacement, stability of the ideal MHD equations is guaranteed. The MHD energy is a very physical quantity which describes the change, caused by distortion of the configurational energy stored in magnetic and thermodynamic energies. An isolated system can only evolve with its total energy (including additional positive kinetic energy) conserved. Hence, if the MHD energy of the configuration increases for every possible distortion, it is clear that such perturbations are prohibited from spontaneously evolving to large amplitudes 
since the system will have more energy than in the equilibrium state. However, if MHD energy is negative upon distortion, it is energetically possible for the system to evolve from small amplitudes, with the excess energy taken up by the kinetic energy of the flow.

\subsubsection{Role of field line curvature}

From the detailed expression for the MHD energy, one can show that the field line curvature plays a major role in determining MHD stability. Consider for example the plasma contained in the magnetic configurations shown in Fig. 4. In Fig. 4a the field lines are convex with respect to the contained plasma, while in Fig. th the field lines are concave. Recall that the curvature is $\boldsymbol{\kappa}=(\mathbf{b} \cdot \boldsymbol{\nabla}) \mathbf{b}$ and $\hat{\mathbf{n}}$ is the normal to the flux surface generated by $\mathbf{B}$, so that $\mathbf{b} \cdot \mathbf{n}=0$. One may also note that if $\mathbf{b} \cdot \boldsymbol{\nabla} \times \mathbf{B}=0$, as is the case of a system with negligible $\beta$, then $\mathrm{b} \times \kappa=\mathrm{b} \times \nabla|B| /|B|$. The MHD energy principle demonstrates that the configuration shown in Fig. $4 \mathrm{a}$ is unfavorable towards stability, while the configuration shown in Fig. $4 \mathrm{~b}$ is highly favorable to stability. For a low beta system this is the same as saying that systems where $|B|$ decreases outwardly are unfavorable to stability, while systems where $|B|$ increases outwardly are favorable to stability. Intuitively, one can argue that a plasma with a pressure, $p$, and mass density, $n M$, has a mean conserved magnetic moment $\mu \approx p / n . M B$, so that the force density on the plasma is $\mathbf{F}=-n \mu \nabla|B|$. For increasing $|B|$, as one moves away from the confined region, there is an inward force which restores plasma perturbations, 
while if $|B|$ decreases outwardly the force is outward and causes perturbations to convect outwardly.

Magnetic mirror machines offer a particularly insightful example of the importance of field line curvature. In a symmetric mirror machine the field line curvature is always unfavorable for stability at the mid-plane. This almost always causes symmetric mirror machines to be MHD unstable except under very restricted conditions.

To obtain a concave-outward field line curvature system, as shown in Fig. $4 b$, one needs to forsake azimuthal symmetry. For example, two axially aligned Helmholtz coils with currents flowing in the same direction can establish a simple mirror machine with unfavorable field line curvature in the mid-plane. Placing four current-carrying rods parallel to the coils' axis but radially displaced and with the currents in the rods alternating in direction, as shown in Fig. 4b, leads to a magnetic field with favorable concave field line curvature properties nearly everywhere.

Experimentally, the entire character of plasma containment in mirror machines was decisively improved once minimum $B$ fields were used that prevented the extremely disruptive curvature driven mode from being excited.

\subsection{Deviation from MHD stability guidelines}

The need to break azimuthal symmetry leads to several technological side

problems such as: stress problems at high fields, difficulty of access (e.g., 
for the injection of neutral beams), increased single particle orbit loss, etc. Hence, considerable interest has remained in devising systems that are basically simple mirrors that maintain azimuthal symmetry. It is conjectured that instability can be attained by creating plasmas that do not obey the dynamics of an ideal MHD system.

\subsubsection{Passive current carriers}

The most common idea is to place an in situ current carrier within the plasma where the current carrier does not respond to the displacements of the plasma. The energetic particle beam in the astron concept is a proposed example of one such current carrier. A passive current carrier can create a min- $B$ field in a symmetric configuration, which can then produce stability in the MHD response of the background plasma. The crucial question is whether the current carrier indeed acts as a passive current carrier and this question needs further study. There are numerous experimental studies in mirror machines and tokamaks that show stability enhancement with energetic particles in the system [e.g. Perkins, et al., 1963; Dandl, et al., 1971; JET Team, 1991]. There are also regimes where new instabilities appear to be caused by the energetic particles.

\subsubsection{Finite Larmor radius stabilization}

Another idea to achieve stability in a symmetric mirror machine, is to exploit the finite orbit size of the bulk plasma ions, which can lead to a plasma 
response that differs from MHD predictions and to so-called finite Larmor radius stabilization [Rosenbluth, et al., 1960]. This mechanism readily stabilizes the shorter wavelength modes, but has difficulty in stabilizing longer wavelength modes. In fact a mode that corresponds to a nearly rigid displacement of the plasma column is extremely difficult to stabilize. This mode can be stabilized at either extremely low densities (far below densities of interest in fusion) or at relatively high beta where currents induced in neighboring conducting walls can serve as a passive feedback mechanism to restore the rigid displacements of the column. At intermediate densities active feedback mechanisms or ponderomotive force from radio frequency fields is needed to achieve stability.

\subsubsection{Migma concept}

An example of a finite Larmor radius concept is the Migma [Maglich, 1988] experiment. Here the machine size is roughly two ion orbits and all the ion orbits pass close to the axis of the machine. In this device, stability at low density has been achieved, but it is below the threshold density predicted for the onset of the unstable displacement mode. Further experiments are needed to test stability for the predictions of finite orbit theory.

\subsection{Stability in Tori}

In toroidal devices MHD stability based on the constraints imposed by field line curvature is very important The stability criteria are somewhat subtle 
to apply. The curvature direction alternates in sign, being unfavorable to stability at major radii $R$ greater than that of the magnetic axis $R_{0}$, while it is favorable to stability at major radii $R$ less than $R_{0}$. The net result depends on the average of the two effects.

\subsubsection{Line average stabilization}

For a low-beta circular tokamak, average favorable curvature arises if the safety factor satisfies $q>1$, while unfavorable average curvature arises if $q<1$. Tokamaks generally operate with most of the flux tubes having

$q>1$. In fact in the center where $q \lesssim 1$, periodic bursts of instability is usually observed, so-called sawtooth oscillations. Though these instabilities are driven by the unfavorable curvature, ideal MHD theory usually does not properly predict these instabilities. They are usually a manifestation of resistive instability, which we shall shortly explain.

\subsubsection{Beta stability limit}

When both good and bad curvature exist on a field line, unstable perturbations try to localize in the bad curvature regions. This causes the field lines to bend, which according to the energy principle induces a stabilizing "bending energy." At sufficiently low beta, ideal MHD theory predicts stability for a toroidal system since the destabilizing energy perturbation driven by the unfavorable curvature cannot compensate the bending energy. Only above a critical beta value. $3_{c}$, can a perturbation be localized to the bad 
curvature region and overcome the stabilizing bending energy. The result is that overall stable operation is limited to beta values below $\beta_{c}$, commonly called the Troyon limit [Troyon, et al., 1984], which is typically $\beta_{c} \approx 2 I_{n}$ (with $I_{n}=\mu_{0} I_{p} / a B_{\phi}, I_{p}$ the plasma current, $a$ the minor radius, and $B_{\phi}$ the toroidal field).

\subsubsection{RFP stability}

If a perturbation has a given pitch along the torus, this pitch can be identical to the rotational transform on one magnetic surface. On that surface there is no bending energy produced by the perturbation. However, if there is magnetic shear, i.e. $q$ varies with the flux surface $\psi$ (i.e. $\partial q / \partial \psi \neq 0$ ), bending energy is produced on neighboring field lines which allows for a stable MHD response for beta values below the appropriate critical $\beta_{c}$. Only if there is a flux tube where $\partial q / \partial \psi=0$ can MHD instability occur at extremely low beta. In pinches, where $q \ll 1$ there will be a surface where $\partial q / \partial \psi=0$ if the toroidal magnetic field does not reverse and this region is a source of virulent instability. When toroidal field reversal is achieved, experimentally there is a dramatic decrease in the magnetic fluctuation levels. As a result the reversed field pinch (RFP), where toroidal field reverses direction inside the plasma, is considered the most interesting low- $q$ toroidal configuration. 


\subsection{Resistive Stability}

If plasma displacements can be localized to bad curvature regions without inducing the stabilizing bending energy then curvature-driven modes can be excited at low beta values. In ideal MHD theory, the ideal Ohm's law, $\mathbf{v} \times \mathbf{B}+\mathbf{E}=0$, with $\mathbf{v}$ the plasma flow speed, forces stabilizing bending energy to be induced when a displacement is localized on a flux tube. With finite resistivity, $\eta$, the Ohm's law is altered to

$$
\mathbf{v} \times \mathbf{B}+\mathbf{E}=\eta \mathbf{J}
$$

with $\mathbf{J}$ the plasma current. The finite resistivity then allows plasma to slip through magnetic field lines. As a result the plasma displacement can be localized to the unfavorable curvature region, without inducing stabilizing bending energy [Furth, et al., 1963]. Thus, due to plasma resistivity, instability can arise from the unfavorable curvature even at low beta.

When this mechanism applies instability growth rates are much slower than the growth rates typical of MHD instability. This mechanism gives rise to the sawteeth magnetic signals inside the $q=1$ surface of tokamaks. The instability causes rapid thermal mixing of the plasma inside the $q=1$ surface. However, as the plasma is stable outside the $q=1$ surface, the integrity of the thermal insulation is maintained on the outside of the plasma. In fact the sawtooth oscillations have the favorable feature of preventing high $Z$ impurities from collecting in the center of these discharges which would cause severe energy losses from radiation. 
The RFP is also prone to resistive instability and the level of magnetic fluctuations is much larger in the RFP than in the tokamak. Here stability tends to be caused throughout the plasma as $q$ is much less than unity everywhere. There is a concern whether the thermal insulation properties needed for fusion can ultimately be achieved with this instability drive present.

In addition to the curvature instability drive, the current profile itself is a source of instability. Here again resistivity allows the current profile drive to manifest itself at low beta, whereas MHD theory would predict stability. In RFP plasmas these current drive instabilities force the configuration to relax close to the luwest energy state consistent with the amount of magnetic flux (or as it is frequently called, magnetic helicity) pumped into the system. This state is one with toroidal field reversed in the inside of the plasma relative to the outside, and it is maintained indefinitely with a fixed amount of in put voltage. Simple diffusion theory for a symmetric equilibrium predicts that a reversed field state cannot be continuously maintained. However, a 3-dimensional dynamo-like effect that is driven by magnetic turbulence is what allows the maintenance of a reversed toroidal field [Taylor, 1986].

\subsection{Microinstability}

Another class of instabilities of importance, quite different from MHD driven instabilities, are referred to as microinstabilities. They generally are of short wavelength (compared to the system size) and their fluctuations do not gen- 
erally lead to catastrophic disassembly, but rather to an enhancement of transport. Even in MHD favorable systems, it is the microinstabilities that often determine the intrinsic containment time of a plasma system.

A homogeneous plasma at a fixed global temperature $T$, is of course in thermodynamic equilibrium and is free of instability. Any departure from thermodynamic equilibrium leads to intrinsic "free energy" drives that have the potential to cause instability and thereby to cause relaxation to the thermodynamic equilibrium state at a rate more rapid than predicted by binary collisions.

\subsubsection{Velocity space instability}

The farther away a system is from thermodynamic equilibrium, the more virulent the drive of the free energy. In simple mirror machines where the ion kinetic distribution is not Maxwellian, there is a velocity space containment condition that must be satisfied, which is given by Eq. (24). As a consequence the distribution function will have a "inverted" population of particles such that the phase space density of low energy particles is less than that of higher energy particles. Such a situation gives rise to a laser-like instability (generally called loss cone instability) in which the inverted distribution tries to relax through coherent oscillations (generally at harmonics of the ion cyclotron frequency) to a "monotonic" state where the phase space density is a decreasing function of energy. However, as the low energy particles are not contained, rapid loss arises on the scale of the particle bounce time. 
In order to sustain a simple mirror plasma it was found to be necessary to supply low energy particles, either through a gas feed or through a low energy particle gun. In the $2 \mathrm{X}$-IIB experiment it was found that with these techniques it was possible to achieve a high beta steady state plasma. Particles were injected at high energy and were contained for a classical slowing down time. The low energy phase space region was filled primarily with plasma arising from the external source, which either prevented instability, or caused a low enough fluctuation level that heated slightly the low energy source but did not greatly perturb the energy containment properties of the high energy particles. The limitation of this method was that the electron temperature was constrained to remain low (typically $T_{e} / T_{i} \approx 0.01$ with $T_{e} \lesssim 100 \mathrm{eV}$ ) and the high ion energy lifetime could not exceed several milliseconds.

One of the pleasing features of the tandem mirror configuration is that the loss cone instability can be prevented. In the center of a tandem mirror the ious do not have a loss cone because these particles are contained by the electric field. In the end cells the loss cone drive is quenched by the outflow of particles from the center. As a result tandem mirrors routinely operate without loss cone instability.

\subsubsection{Drift wave instability}

In toroidal plasmas one is generally not concerned with velocity space instabilities. However, as intrinsic temperature and density gradients must exist to thermally isolate the system from the walls, the system cannot be 
in global thermodynamic equilibrium. The intrinsic gradients give rise to a diamagnetic flow rate which from the equilibrium equations are

$$
\mathbf{J}_{j}=\frac{\mathbf{B} \times \nabla p_{j}}{B^{2}} \equiv e_{j} n_{j} v_{D_{j}}
$$

where the species $j$ can refer to electrons or ions and $v_{D j}$ is diamagnetic drift frequency of species $j$. In a frame moving at the equilibrium $\mathbf{E} \times \mathbf{B}$ speed, $v_{D e}$ and $v_{D i}$ would be the mean flow speed of electrons and ions respectively. These flows are in opposite directions and it is impossible to find a reference frame where the overall flow of every specie can be ignored. These equilibrium flows are the free energy source for instability. If we consider a perturbation with a spatial dependence proportional to $\exp (i \mathbf{k} \cdot \mathbf{r})$, a low frequency response will arise near the angular frequency $\omega \sim \mathbf{k} \cdot v_{D_{j}}$. It is found that when instability arises at this frequency, it causes anomalous relaxation of th. plasma. Often the magnitude of the growth rate $\gamma$ approaches that of the real frequency.

\subsection{Turbulent Diffusion}

The rate of diffusion caused by drift instabilities can be estimated by the following mixing length argument. A wave with an electric field amplitude $\delta E$ will grow until the $\mathbf{E} \times \mathbf{B}$ drift velocity of the wave forces the plasma to displace a wavelength in a growth time, i.e.

$$
\delta \mathbf{r} \simeq \frac{\delta \mathbf{E} \times \mathbf{b}}{\gamma B} \approx \frac{1}{k}
$$


where $\delta \mathbf{r}$ is the plasma displacement by the wave. The resulting wave breaking then forces saturation. Thus, we have a random motion $\delta r \sim 1 / k$ in a correlation time $\tau=1 / \gamma \approx 1 / k v_{D_{j}}$. Taking the diffusion coefficient to be $D_{\text {anom }} \approx \delta r^{2} / \tau$, gives

$$
D_{\text {anom }} \approx \frac{\gamma}{k^{2}} \approx \frac{1}{\epsilon_{j} k B n} \frac{\partial\left(n T_{j}\right)}{\partial r} .
$$

If $k \sim 1 / r$, this diffusion coefficient yields so-called Bohm diffusion (taking $\left.r \frac{\partial}{\partial r} \sim 1\right)$

$$
D_{\text {Bohm }} \simeq \frac{T_{j}}{e B}
$$

Such diffusion produced by drift waves is possible in a shearless system. In tokamaks and other toroidal devices, the magnetic shear often forces the wave number to scale as the ion Larmor radius $\rho_{i}$, giving rise to a modified Bchm scaling

$$
D_{M B} \approx \frac{T_{j}}{e B} \frac{\rho_{i}}{L_{p}}
$$

with $L_{p}^{-1}=\nabla\left|n T_{j}\right| / n T_{j}$.

It appears that tokamak data is roughly consistent with modified Bohm scaling. However, the interplay among the number of degrees of freedom is sufficiently complicated that a definitive description of turbulent transport still needs to be developed and it is currently an extremely active research area [Callen, 1990]. 


\section{STATUS OF MAGNETIC FUSION RESEARCH}

\subsection{Historical Overview}

The quest to achieve controlled fusion developed soon after the successful development of the hydrogen bomb in the 19.50s. The rapid success of new scientific technology, including fission power, produced great optimism that controlled fusion could be achieved relatively quickly. However, the insta" bility problems in plasma containment were not fully appreciated. Indeed many research groups initially inferred the achievement of thermonuclear conditions when neutrons were produced in experiments. It usually turned out that these neutrons resulted from runaway ions produced by the large electric fields associated with the turbulent disruption of the plasma.

By the early 1960s a general pessimism pervaded the program, as plasma confinement in nearly all devices was poor, with confinement properties often being attributed to Bohm diffusion. During this difficult period the subtleties needed to achieve good confinement began to be appreciated.

Major breakthroughs developed, often due to the understanding of "kitchen" physics, i.e. the solving of problems associated with wall conditioning, impurity control, vacuum conditions, etc. and due to the understanding of how to achieve configurations that would be stable to gross instabilities. Experimental results demonstrated relatively long-lived containment in a variety of concepts. The experimental concep: that received the greatest ap- 
preciation was the tokamak, which has become the leading magnetic fusion concept and is at present, the most developed. There have been important breakthroughs in other concepts as well, including: mirror machines, RFP, compact toroid, and stellarators.

$\mathrm{By}^{\prime}$ the mid 1970s the success of multiple approaches to achieving fusion and the appreciation of the need to develop a long-term energy source, lead to a broad support by the U.S. guvernment of many approaches in magnetic fusion. However, budgetary constraints became important in the United States fusion program in the $1980 \mathrm{~s}$. As a result, most of the research programs not connected to tokamaks, were terminated, often before scientific issues were clarified. Thus, crucial questions in ascertaining the potential usefulness of many of the approaches are unanswered. In some cases they can be addressed in fusion programs outside the United States, where significant research facilities exist in stellarators, RFP and mirror machines.

To present an overview of progress in magnetic fusion in somewhat more detail, we shall give a brief status report of the approaches that are alternate to tokamaks and then present a more detailed status report of the tokamak concept. 


\subsection{Status of Non-Tokamak Concepts}

\subsubsection{Mirror machines}

Initial mirror machines experiments were in symmetric mirror fields, and if the density was not too low, they demonstrated poor confinement due to the destabilizing curvature driven instability. A major breakthrough occurred [Ioffe, et al., 1964] when it was demonstrated that the placement of quadrupole coils to form an MHD stable min- $B$ configuration, resulted in an order of magnitude increase in the containment time.

The next major breakthrough in mirror research was the demonstration that high beta plasma can be sustained by neutral beam injection [Coensgen, et al., 1976]. This experiment also demonstrated the importance of neutral beam technology, which was rapidly applied to tokamak and stellarator research.

An apreciation, that the energy gain of a mirror machine under fusion conditions needed to be improved, then led to the invention of the tandem mirror concept [Dimov, et al., 1976; Fowler, et al., 1977]. The experimental group at the Livermore Laboratory demonstrated feasibility of the tandem mirror and in particular showed that loss cone instability can be completely suppressed as first suggested by a theory [Post, et al., 1966]. The attempt to improve the tandem mirror concept by creating thermal barriers proved to be illusive. At present the (iAMMA-10 tandem mirror, the only tandem mirror which is an operation in 1992. in Tskubo. Japan has shown that at 
relatively low density that quiescent long-lived tandem mirror plasmas can be obtained with thermal barrier operation [Miyoshi, et al., 1992]. The major improvement needed is to increase the operational density and the life time of the thermal barrier.

Though simple mirror machines have marginal applicability as an energy source, they may have a special uniqueness for application as a $14 \mathrm{MeV}$ neutron source which will be needed for testing the properties of materials that surround a fusion reactor [Berk, et al., 1990]. A d-t plasma in a mirror machine can supply neutron intensity to achieve a needed fluence in the shortest possible time and at relatively low cost, because a high beta (which allows a high intensity), and small volume (which reduces overall cost) plasma can be achieved. Currently, studies of this application are ongoing in several laboratories, and may lead to a resurrection of interest in mirror machine research.

\subsubsection{Reversed field pinch}

Many different high beta pinch concepts were examined in the 1960s. The most successful ultimately turned out to be the Zeta experiment (which originally was designed as a stabilized $z$-pinch) where a quiescent operational region was observed [Robinson. 1969]. In this regime the toroidal magnetic field in the center was reversed from its direction at the edge. Surprisingly, the reversal could be sustained by toroidal voltage, despite the prediction of two-dimensional diffusion theory that it would not be sustained. 
Though the Zeta experiment was terminated, perhaps prematurely in light of its discovery, the reversed field pinch configuration has been now studied in many facilities worldwide. These experiments have demonstrated reduced fluctuation level and improved containment with increasing temperature. These results are consistent with the theoretical understanding that the plasma will try to relax towards its lowest energy state and the relaxation process allows a steady plasma to be maintained through turbulent dynamo processes. In principle this mechanism can be extended to achieve steady operation with non-Ohmic helicity injection.

Though magnetic fluctuations in an RFP are low cornpared to most other high beta pinches, the fluctuations are appreciably higher than in tokamaks, and are present close to the plasma edge. Such fluctuations lead to impurity buildup, heat loading problems, and the penetration of magnetic fields into the walls which in turn enhances fluctuations. It is foreseen that by increasing the current and temperature of an RFP, the fluctuation level will decrease, as the rate of relaxation needed to remain close to the lowest energy state is reduced. The verification of this conjecture is the principal goal of the European group in Padua, Italy, who are presently building a large machine that will have a plasma current of 4 Megamps [Antoni, et al., 1989].

\subsubsection{Compact toroids}

Another off-shoot of pinch research was the study of compact toroids. Early it was found that by programming the wall currents in a theta pinch ex- 
periment, the $\hat{z}$-directed magnetic fields could be reversed into an elongated compact toroid without any apparent toroidal magnetic field. However, typically, these experiments would disrupt relatively rapidly due to the onset of a rotational instability. By appropriate preparation it was demonstrated [Eskov, et al., 1979] that elongated field reversed theta pinches can be made free of the rotational instability. After a great deal of study these results were confirmed by other experimental teams. In the 1980s it was shown in Japan that min- $B$ quadrupole fields could be used to insure MHD stability [Ishimura, et al., 1989].

An alternative approach to compact toroids started in the late 1970 s with the study of spheromaks, a compact toroid which has both toroidal and poloidal magnetic fields. Several novel ideas were developed to form plasmas using pulsed techniques. Both the poloidal and toroidal magnetic flux needs to be embedded in the plasma during its formation phase, and then the plasina has to be guided into a storage region where it is contained. In the more successful experiments a relatively high electron temperature was attained, $T_{e}>100 \mathrm{eV}$ [Yamada, et al., 1989], and external magnetic helicity injection was used to sustain the configuration [Jarboe, et al., 1983].

Perhaps the first compact toroid conceived was the astron concept [Christopholis, 1962]. Here field reversal is to be achieved with the current produced by energetic particles whose Larmor radius is comparable to the radius of the plasma. The demonstration field reversal has been achieved 
[Andrews, et al, 1971], but there is still difficulty in achieving hot plasma containment in an astron configuration.

One possible future direction in compact toroid research is to merge the astron and field reversed theta pinch concepts. The joint system should stabilize the so-called tilt instability [Rosenbluth, et al., 1979], an MHD instability that might otherwise prevent the achievement of reversed field theta pinch devices with relatively small Larmor radii.

Finally, it should be noted that the reversed field theta pinch is an intrinsically high beta device. If suitable plasma confinement can be successfully achieved the configuration would be especially suitable for advanced fuel cycles, such as $\mathrm{d} \cdot{ }^{3} \mathrm{He}$, since synchrotron radiation losses would be insignificant and the fusion power production density would be high, while the total power production would be moderate due to the system's compactness [Kernbichler, et al., 1992].

\subsubsection{Stellarators}

Progress in stellarators was initially very slow, in part because of the complications in designing 3-dimensional magnetic fields. For example, it was found that stellarators were frequently built improperly without magnetic flux surfaces existing. Careful field design by the research team at the Max Planck Institute lead to more favorable stellarator containment by the late 1960s [Berkl, et al., 1969]. Further innovations by the Kyoto group in Japan were able to show that plasma containment in stellarators is comparable to similar 
size tokamaks [Uo, et al., 1977]. With these studies the research teams found that by heating with r.f. field or with high energy neutral beams, confinement times could be achieved that were similar to that in comparable size tokamaks. Further, it was shown the radial electric field could be controlled to significantly improve particle containment, even those of the injected high energy particles.

The stellarator has considerable flexibility of design, and indeed new interesting designs emerge every few years. Some of the more interesting magnetic field design concepts that have emerged are [Grieger, et al., 1992]: modular magnetic coils which allow for ready disassembly for maintenance; magnetic field design that can minimize the particle excursion from a flux tube; produce large stabilizing magnetic shear; produce favorable min- $B$ properties, etc.

Stellarators are now the principal backup to tokamaks. The often-cited advantages of stellarators over tokamaks are that stellaratiors have been free from the catastrophic disruptive loss to which tokamaks are susceptible and that there is no need for an elaborate current drive mechanism. The complexity of a siellarator is also its virtue as the optimum stellarator is probably yet to be designed. A large stellarator (LHD) is now under construction in Nagoya, Japan, and plans for a large stellarator exist in Garching, Germany. 


\subsection{Status of Tokamak Research}

Tokamak research achieved a major breakthrough in 1968 when a favorable regime operation was reported [Artsimovich, et al., 1968]. The favorable regime was rather narrow, with too strong a current producing violent MHD instabilities since $q<1$ on axis, whereas too weak a current could not produce enough power to sustain the discharge. The discovery of a window of favorable operation truly changed the direction of controlled fusion research. Many research groups throughout the world converted to tokamak research.

A great deal of progress has occurred since the initial breakthrough was reported in 1968. Some of the crucial developments are described below.

\subsubsection{Impurity control and fueling}

Impurity control of the discharge has improved greatly by the development of wall preparation and fueling techniques. Initially tokamaks operated with a high $Z_{\text {eff }}(\sim 5-10)$, whereas presently $Z_{\text {eff }}<2$ is now standard in tokamak operation. Recently, technology has been developed for injecting pellets that allows for central fueling which often leads to substantially improved confinement characteristics at low $Z_{\text {eff }}$. However, the issue of whether impurity build-up can be prevented over extremely long pulse times $(>10 \mathrm{sec}$ ) and under high heating power, remains crucial and will be investigated in future experiments. 


\subsubsection{Auxiliary heating}

Auxiliary heating is generally necessary at high temperature as ohmic heating loses its effectiveness because, at fixed current, the heating rate is proportional to $T_{e}^{-3 / 2}$. Only if large plasma current densities are present, which can occur in high field machines with $B_{\phi}>10$ Tesla, is it conceivable that ohmic heating can bring a plasma to ignition conditions. The advances in neutral beam and radio frequency technologies have enabled the achievement of the needed plasma temperature for producing fusion. For example, the TFTR experiment at Princeton, New Jersey, and the JET experiment in Culham, England have produced plasmas with ion temperatures in excess of $20 \mathrm{keV}$ and electron temperatures of $\sim 10 \mathrm{keV}$. In the TFTR experiment the best confinement characteristics are obtained in the "supershot" regime [Bell, et al., 1989], where neutral beams directly fuel the center of the discharge. Neutral beams have also been used in TFTR to simulate collective alpha particle effects of a fusion-producing plasma [Wong, et al., 1991; Heidbrink, et al., 1991]. In the JET experiment, high energy tails have been generated with ion cyclotron heating that simulate the effect of charged energetic fusion products in the plasma [JET Team, 1991]. In this experiment sawteeth instability is eliminated, perhaps as a result of hot particle stabilization of MHD instability. 


\subsubsection{Plasma shaping}

The shaping of a tokamak plasma has become a crucial feature in tokamak performance. The initial tokamak experiments were with circular-shaped poloidal cross-sections which lead to a relatively low critical beta values for the onset of MHD stability. By means of plasma shaping, MHD stability properties can be substantially improved. Recently the D-III-D tokamak, a shaped tokamak at General Atomic in San Diego, has achieved peak beta values of $40 \%$ and an average beta value of $\sim 10 \%$ [Lazarus, et al., 1991]. It is believed that this plasma can test the so-called second stability regimes [Coppi, et al., 1979], where stability can be attained in a tokamak even at high beta. If such a high beta stability regime can indeed be achieved it makes $\mathrm{d}-{ }^{3} \mathrm{He}$ operation feasible in a tokamak.

In addition, with shaping it is easier to cause field lines near the edge to be deliberately diverted to the wall (such field line design is called a divertor). The purpose is to decouple the properties of the plasma-wall interactions from the properties of the confined plasma and thereby prevent impurity buildup in the plasma although impurities are being generated at the wall. In addition, it is found that divertor operation facilitates $\mathrm{H}$-mode operation, where the plasma develops a sharp pressure gradient at the edge and the overall plasma confinement increases by a factor of $\sim 2-3$ from the usual operating scenario [Jackson, et al., 1992].

Another type of shaping is that of the current profile. It has recently 
been observed [Zarnstorff, et al., 1991] that there is better confinement if the current profile is altered from the natural profile reached in steady operation. Techniques to develop favorable current profiles in steady state need to be developed.

\subsubsection{Current drive}

Non-inductive current drive techniques have made considerable progress. Tokamak currents have successfully been driven by r.f. sources (at lower hybrid and electron cyclotron frequencies) and by neutral beams. At low plasma currents, these current drive mechanisms have allowed long time plasma operation [Jacquinot, et al., 1992] in many large and small machines. However, these current drive schemes are intrinsically less efficient than the inductive (ohmic) drive methods and the efficiency decreases at higher densities. In reactor operation at relatively high beta, the requirements of current drive are lowered by the large bootstrap current induced and it is conceivable that an acceptable current drive efficiency can be achieved [Cordey, 1988].

In addition to the above mentioned current drive mechanisms there are alternate ideas being investigated, such as magnetic helicity injection [Jarboe, 1983], that was first studied in RFP research.

Without current drive the duty cycle of a tokamak reactor needs to be high and thermal stresses, due to wall cooling and heating as the tokamak is turned on and off, need to be minimized. The JET facility ha recently shown that the plasma current can be reversed by A.C. cycling of the ohmic 
drive [Tubbing, 1992]. They demonstrated that the time interval in which no plasma equilibrium exists can be short $\sim 1 \mathrm{sec}$. This mode of operation can conceivably mitigate the heat stress problem.

\subsubsection{Achieved confinement parameters}

Finally, we note that the progress in tokamak containment can be summarized in Fig. 13, which presents values of the parameter, $n \tau T$, which have been achieved in various tokamaks during the last two decades. One observes that the quality of plasma confinement now being achieved in tokamaks is nearly equivalent to achieving energy breakeven with the $\mathrm{d}$-t fusion reaction. In addition, the JET facility [JET Team, 1992] has now actually operated with d-t, producing $Q \sim .15$ in a lean d-t mixture $(Q \sim .45$ would have been produced in an optimum $\mathrm{d}$-t mixture in the same experiment; in addition, plasma confinement conditions that would have produced $Q>1$ in an optimum d-t mixture was produced in a d-d discharge). Similar runs are being planned for TFTR. An international collaboration, ITER, is now being formed to design and build a tokamak that will achieve ignition and exhibit prolonged operation with fusion produced alpha particles [Tomabechi, et al., 1991].

\subsection{Conclusion}

The advances in tokamak research has reached the point that the next major step is the building of an ignition experiment and possibly to study the 
system during the burn phase. It is proposed that this experiment be run by an international collaboration, which is now called ITER. In addition, work on smaller size tokamaks is necessary to better understand physical phenomena and find ways that the intrinsic operation in tokamaks can be improved. As the advances in tokamak have often occurred in unplanned areas, substantial improvements can certainly be expected.

Research in alternate confinement schemes have not had the sal... .upport as tokamaks and it is not surprising that their achievements lag behind the tokamak. Some of these schemes may evolve to reactor grade concepts, while others might be highly favorable for non-energy producing applications such as: a compact neutron source for material testing or a compact source for producing medically desired radioactive isotopes. Research in these concepts have frequently contributed significantly to the overall progress in the physics of plasma containment or to the generation of technological tools. Hence, the controlled fusion program will continue to profit by continuing its support of alternate directions of research. 


\section{GLOSSARY}

ambipolar potential: The electrostatir potential a piasma establishes for maintaining overall charge neutrality.

anomalous transport: The transport in a plasma due to self-generated electromagnetic fuctuations.

banana orbit: The shape of an orbit of particles in a tokamak which are trapped by the increasing magnetic field.

beta: The ratio of kinetic pressure of a plasma to the magnetic field pressure.

classical transport: A theoretical description of transport of a plasma in a uniform magnetic field.

compact toroid: A confinement concept of a toroidal plasma without magnetic field coils through its center.

Lawson criterion: The product of density and plasma lifetime which is a parameter that measures the quality of plasma confinement in regard to what is needed to achieve fusinn corditions.

magnetic flux surfaces: The surface on which magnetic field lines lie in a toroidal confinement concept.

Magnetonydrodynamics (MHD): The fluir description of ionized gases.

MHD energy: The jerturbed energy of a system described by the MHD equation.: 
mirror machine: A confinement concept consisting of open-ended magnetic field lines with the magnetic field intensity increasing towards the ends.

neoclassical transport: A collisional transport theory that is based on the finite orbit size due to drift orbit excursions in tokamaks and other plasma confinement machines.

pinch: A generic plasma confinement configuration with large plasma currents.

plasma: lonized gas

reversed field pinch: A pinch device where the toroidally directed field reverses between the plasma center and the edge.

rotational transform: The number of times a field line winds around the poloidal cruss-section of a torus for each time it winds arounu the toroidal cross-section.

safety factor $(q)$ : The inverse of the rotational transform; $q>1$ generally indicates stability in tokamaks, while $q<1$ indicates a likelihood of instability.

stellarator: A three-dimensional magnetic configuration that does not require large plasma currents to achieve plasma containment.

tokamak: A pinch-like configuration with a strong toroidal magnetic field and a strong toroidal current carried by the plasma. 


\section{List of Works Cited}

Andrews, M.L., Daviton, H., Fleischmann, H., et al. (1971), Physical Review Letters 27. $1+28$.

Antoni, V., Bagatin, M., Baseggio, E., et al., Plasma Physics and Controlled Vuclear Fusion Research 1988, Vol. 1 (IAEA, Vienna, 1989) p. 661 .

Artsimovich, L.A., Bobrovskii, G.A., Gorbonuv, E.P., et al., Plasma Physics and Controlled Nuclear Fusion Research, Vol. I (IAEA, Vienna, 1969) p. 157.

Asdex Team (1989), Vuclear Fusion 29, 1959.

Bell, M.G., Arunasalam, V., Barnes, C.W., et al., Plasma Physics and Controlled Nuclear Fusion Research 1988, Vol. 1 (IAEA, Vienna, 1989) p. 27.

Berk, H.L., and Ryutov, D.D. (1990), Comments Plasma Physics and Controlled Fusion $13,173$.

Berkl, E., Eckhartt, D., Gierke, G.V., et al., Plasma Physics and Controlled Nuclear Fusion Research 1908, Vol. I (IAEA, Vienna, 1969) p. 513.

Callen, J.D. (1990). Physics of Fluids B 2, 2869, and related articles in this issue.

Callen, J.D. (1992). Physics of Fluids B 4. 2142.

('arreras, B.A.. Cireiger, G., Harris, J.H., et al. (1988), Nuclear 
Fusion 28, 1613.

Christofilis, N.C. (1962), Nuclear Fusion Supplement, Part 1, 159.

Coensgen, F.H., Cummins, W.F., Gormezano, C., et al. (1976), Physical Review Letters 37, 143.

Coppi, B., Ferreira, A., Mark, M.W.K., and Ramos, J.J. (1979), Nuclear Fusion 19, 715.

Cordey, J.G. (1988), Plasma Physics and Controlled Fusion 30,1625 .

Dandl, R.A., Eason, H.O., Edmonds, P.H., and England, A.C. (1971), Nuclear Fusion 11, 411.

Dimov, G.I., Zakaidakov, V.V., Kishinevshii, M.E. (1976), Fiz. Plasmy 2, 597.

Drake, J.R., Brunsell, P., Brzozowski, J., et al., Plasma Physics and Controlled Nuclear Fusion Research 1988, Vol. 2 (IAEA, Vienna, 1989) p. 751 .

Es'kov, A.G., Kurtullaev, R. Ku., Kreshchuk, A.P., et al., Plasma Physics and Controlled Nuclear Fusion Research 1978, Vol. II (IAEA, Vienna, 1979) p. 187.

Fisch, N.J. (1987), Reviews of Modern Physics 59, 175.

Fowler, T.K., and Logan, B.G. (1977), Comments Plasma Physics and Controlled Vuclear Fusion Research 2, 167. 
Freidberg, J.P. (1982), Reviews of . Modern Physics 54, 801.

Furth. H.P., Killeen, J., and Rosenbluth, M.N. (1961), Physics of Fluids 6, 459 .

Galeev, A.A., and Sagdeev, R.Z., Reviews of Modern Physics 7 Ed. M.A. Leontovich, (Consultants Bureau, New York, 1979) p. 1 .

Grieger, (i., Lotz. W., Merkel, P., et al. (1992), Physics of Fluids $B$ 4, 2081.

Haines, M.G., Dangor, A.E., Choi, P., et al., Plasma Physics and Controlled Vuclear Fusion Research 1990, Vol. 2 (IAEA, Vienna, 1991) p. 769.

Heidbrink, W.W., Strait, E.J., Doyle, E., et al. (1991), Nuclear Fusion 31, 1635.

Ioffe, M.C., and Sobolev, R.I. (1964), Atomic Energy 17, 366.

Ishimura, T., Veda, Y., S.gimoto, S., et al., Plasma Physics and Controlled Vuclear Fusion Research 1988, Vol. 2 (IAEA, Vienna, 1989) p. 705.

Jackson, G.L., Winter, J., Taylor, T.S., et al. (1982), Physics . of Fluids $B$ 4, 2181 .

Jacquinot, J., Bures, MI, and JET Team (1992), Physics of Fluids $B 4,2111$.

Jarboe, T.R., and Henins, I., Sherwood, A.R. (1983) Physical 
Review Letters 51, 39.

JET Team, Plasma Physics and Controlled Nuclear Fusion Research 1990, Vol. 1 (IAEA, Vienna, 1991) p. 437.

JET Team (1992), Nuclear Fusion 32, 187.

Kernbichler, W., Hendler, M., Momota, H., et al., Plasma Physics and Controlled Vuclear Fusion Research 1990, Vol. 3 (IAEA, Vienna, 1991) p. 555.

Lazarus, E.A., Chu, M.S., Ferron, J.R., et al. (1991), Physics of Fluids $B$ 3, 2220.

Maglich, B. (1988), Nuclear Fusion Methods and Physical Review $A \stackrel{27,13 .}{13}$

Miyoshi, S., Cho, T., and Hojo, H., et al., Plasma Physics and Controlled Nuclear Fusion Research 1990, Vol. 2 (IAEA, Vienna, 1991) p. 539.

Perkins, W.A., and Post, R.F. (1963), Physics of Fluids 6, 1537.

Post, R.F., and Rosenbluth, M.N. (1966), Physics of Fluids 9,730 .

Robinson, D.C., and King, R.E., Plasma Physics and Controlled Nuclear Fusion Research, Vol. I (IAEA, Vienna, 1969) p. 263.

Rosenbluth, M.N., Krall, N.A., Rostoker, N. (1960), Nuclear 
Fusion Supplement, Part 1, 143.

Rosenbluth, M.N., Bussac, M.N. (1979), Nuclear Fusion 19, 489.

Ryutov, D.D. (1975), Usp. Fiz. Nauk 116, 341.

Taylor, J.B. (1986), Reviews of Modern Physics 58, 741.

Tomabechi, K., Gilleland, J.R., Sokolov, Yu.A., et al. (1991), Nuclear Fusion 31, 1135.

Troyan, F., Gruber, R., Saurenmann, H., et al. (1984), Plasma Physics and Controlled Vuclear Fusion 26, 209.

Tubbing, B.J., Gottardi, N.A.C., Green, B.J., et al. (1992), Nuclear Fusion 32, 967.

Uo, K., Iiyoshi, A., Obiki, T., et al., Plasma Physics and Controlled Nuclear Fusion Research 1976, Vol. II (IAEA, Vienna, 1977) p. 103.

Wittenberg, L.J., Santarius, J.E., Kulcinski, G.L. (1986), Fusion Technology 10, 167.

Wong, K.L., Fonck, R.J., Paul, S.F., et al. (1991), Physical Review Letters 66, 1874.

Yamada, M., Ellis, R.A., Jr., Janos, A.C., et al., Plasma Physics and Controlled Vuclear Fusion Research, Vol. 2 (IAEA, Vienna, 1989) p. 539.

Zarnstorff, M.. Barnes, C.W., and Efthimion, P.E., et al., 
Plasma Physics and Controlled Nuclear Fusion Research 1990, Vol. 1 (IAEA, Vienna, 1991) p. 109. 


\section{Figure Captions}

1. The solid curves are reaction rates averaged over a Maxwellian distribution as a function of temperature (in $\mathrm{keV}$ units) and dashed curves are cross-sections as a function of center of mass energy for the d-d, $d-t$, and $d \cdot{ }^{3}$ He fusion reactions.

2. Lawson diagram for sustaining fusion burn for the $\mathrm{d}-\mathrm{d}, \mathrm{d}-\mathrm{t}$, and $\mathrm{d}-{ }^{3} \mathrm{He}$ reactions.

3. Typical toroidal configuration showing coordinates $(Z, R, \phi)$ and $(r, \theta, \phi)$.

4. Two mirror configurations which are examples of open-ended machines. The symmetric mirror (a) tends to be MHD unstable. With a quadrupole field, shown in (b) stabilizing concave-shaped field lines are produced.

5. (a) In the usual toroidal configuration the plasma is surrounded by external toroidal field coils as indicated schematically by the heavy lines with arrows. (b) The plasma region and field coils are shown for the Text tokamak, at The University of Texas, Austin. The central region is the structural support as well as the magnetic flux column that is used as a transformer to drive plasma current

6. Schematic flux surface of a compact toroid. The 3-dimensional configuration is envisaged by evolving this plot around the $z$-axis. Closed poloidal field lines are closed inside the separatrix curve passing through 
the points labelled $x$. Outside the separatrix, field lines are open. The poloidal flux maximizes at the poloidal field null inside the toroid where $B_{\theta}=0$ and which is indicated by the points labelled 0 . There are no external current carriers penetrating the torus. A toroidal field inside the toroid can arise from plasma currents flowing along poloidal field lines.

7. Transformer link to a toroidal plasma. The change of magnetic flux inside the central transformer column supplies toroidal voltage to the plasma.

8. (a) Magnetic surfaces showing separatrix points, and separatrices for $\ell=1,2,3$ and 4 stellarators and for the $\ell=3$ torsatron. (b) Threedimensional view of coil set of an $\ell=2$ classical stellarator (Wendelstein VII-A) showing the twisting magnetic flux surface (from Nuclear Fusion 28, 1613, R. Carreras, et al.)

9. (a) Mction of a charged particle in a uniform magnetic field. (b) Motion of a charged particle in uniform electric and magnetic fields that are perpendicular to each other. The particle guiding center moves in a plane perpendicular to $\mathbf{E}$.

10. (a) Electrostatic potential and (b) magnetic field in a tandem mirror configuration as a function of the distance $s$ along a field line (note that the negative $s$ region is a reflection of the positive $s$ region). $\Phi_{i}$ and $\Phi_{e}$ 
are the containing potentials for ions and electrons. With a thermal barrier present, $\Phi_{b}$ is the potential that isolates electrons in the central cell from the end plug.

11. Particle motion in a tokamak projected onto a fixed $\phi$ cross-section. In (a), particles moving counter to the toroidal current are shifted to orbits that are outside the dotted flux surface, while particles moving with the toroidal current have orbits interior to the flux surface. The orbit of a trapped particle is shown in (b) and (c). In (c) the orbit is displaced due to a toroidal electric field, giving rise to an overall inward drift motion.

12. Potential diagram for one-dimensional motion.

13. Fusion parameter $n T_{E} T_{i}$ versus central ion temperature $T_{i}(0)$ for a number of machines worldwide in the period 1965-1991 (figure published with permission from JET Joint Undertaking, which retains copyright of the material). 


\section{Further Reading}

Handbook of Plasma Physics; Basic Plasma Physics I, II (1983), Editors A.A. Galeev and R.N. Sudan, Amsterdam: North-Holland.

Fusion, Volume I: Magnetic Confinement (1981), Edited by Edward Teller, New York: Academic Press.

"The Magnetic Mirror Approach to Fusion," R.F. Post, Nuclear Fusion 27, $1579(1987)$

Nuclear Fusion (1990), Vol. 30, p. 1637-1996 (this issue contains review papers on all the principle approaches to Magnetic Fusion). 


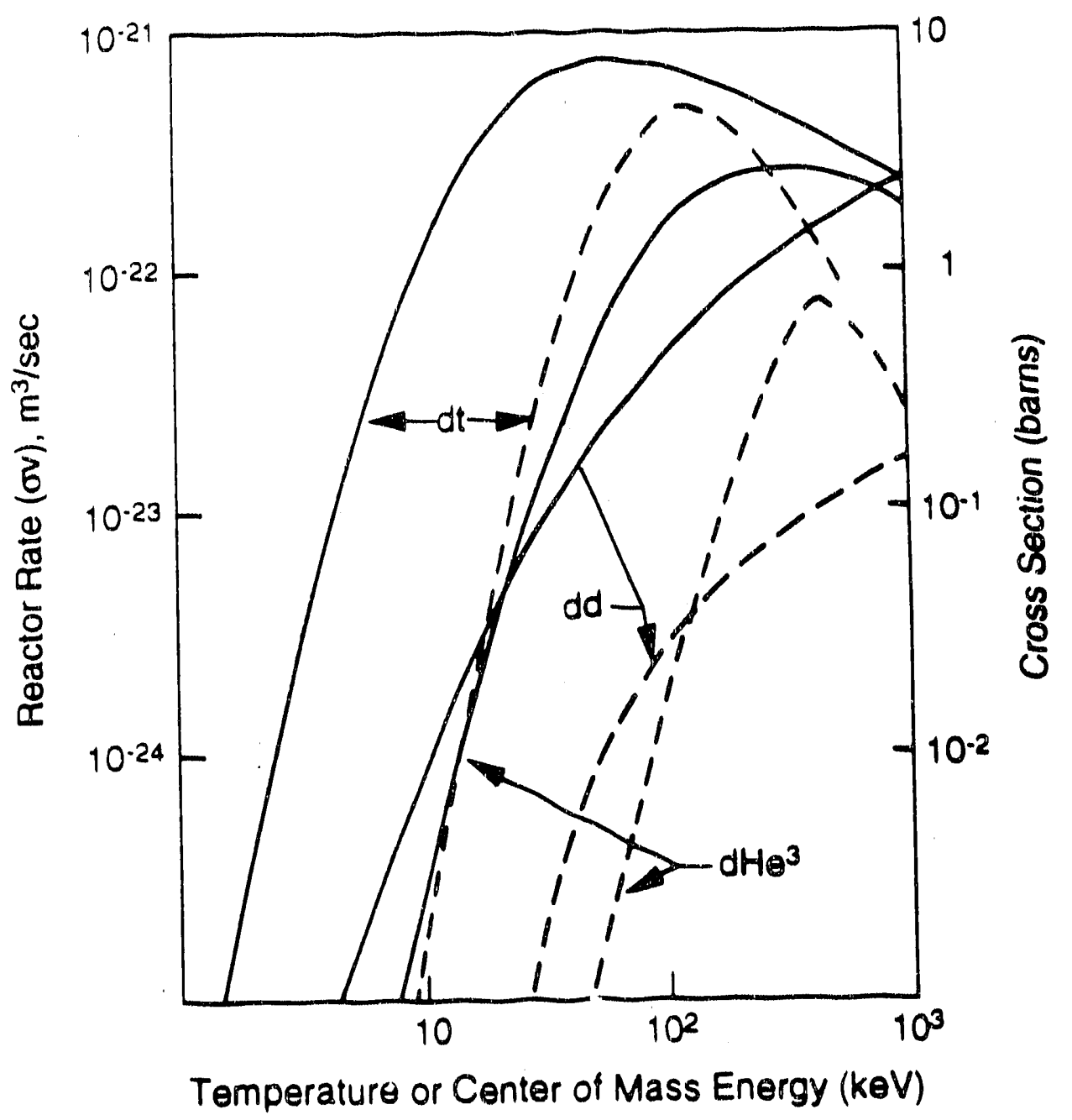

Fig. 1 


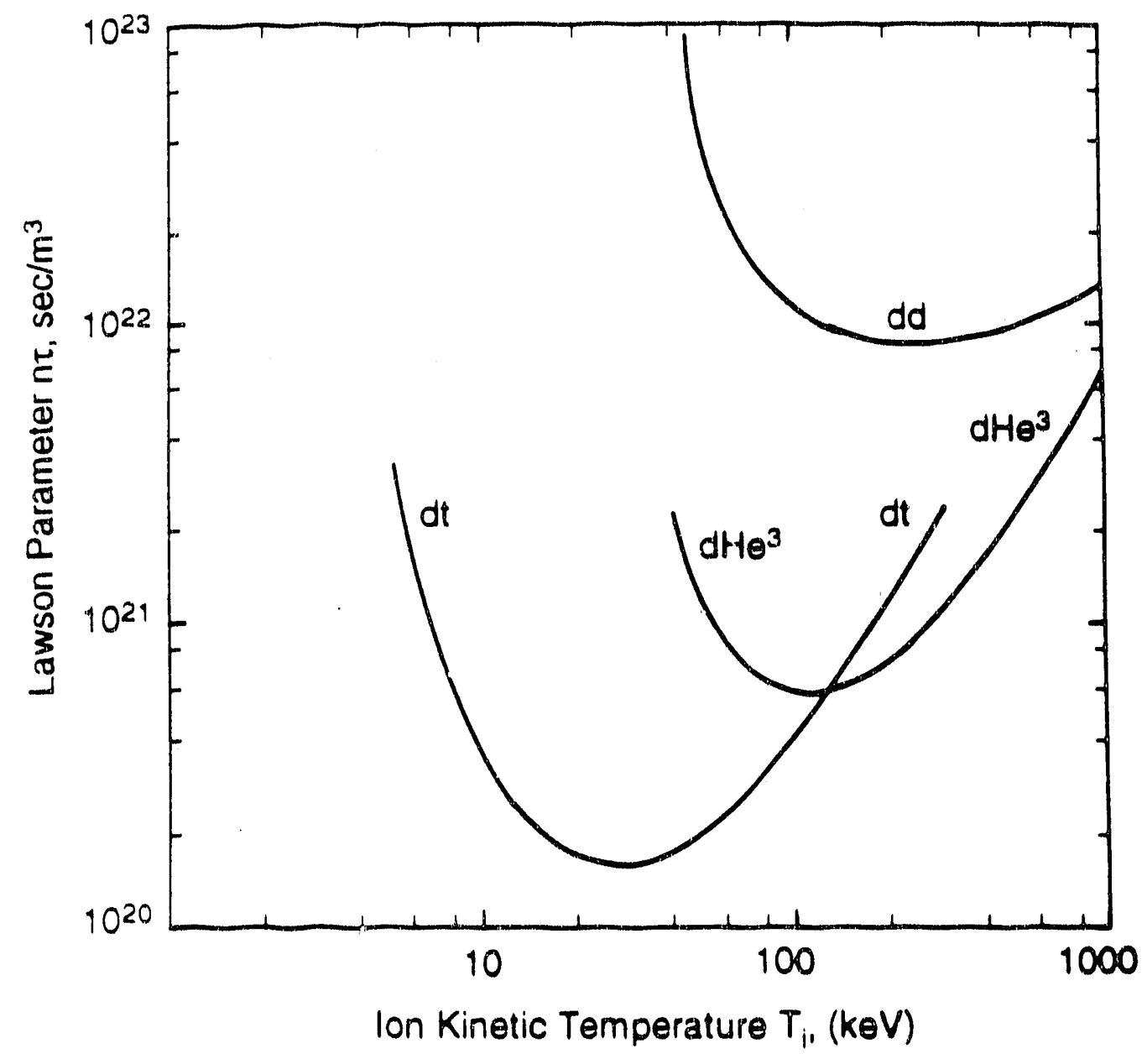

Fig. ? 


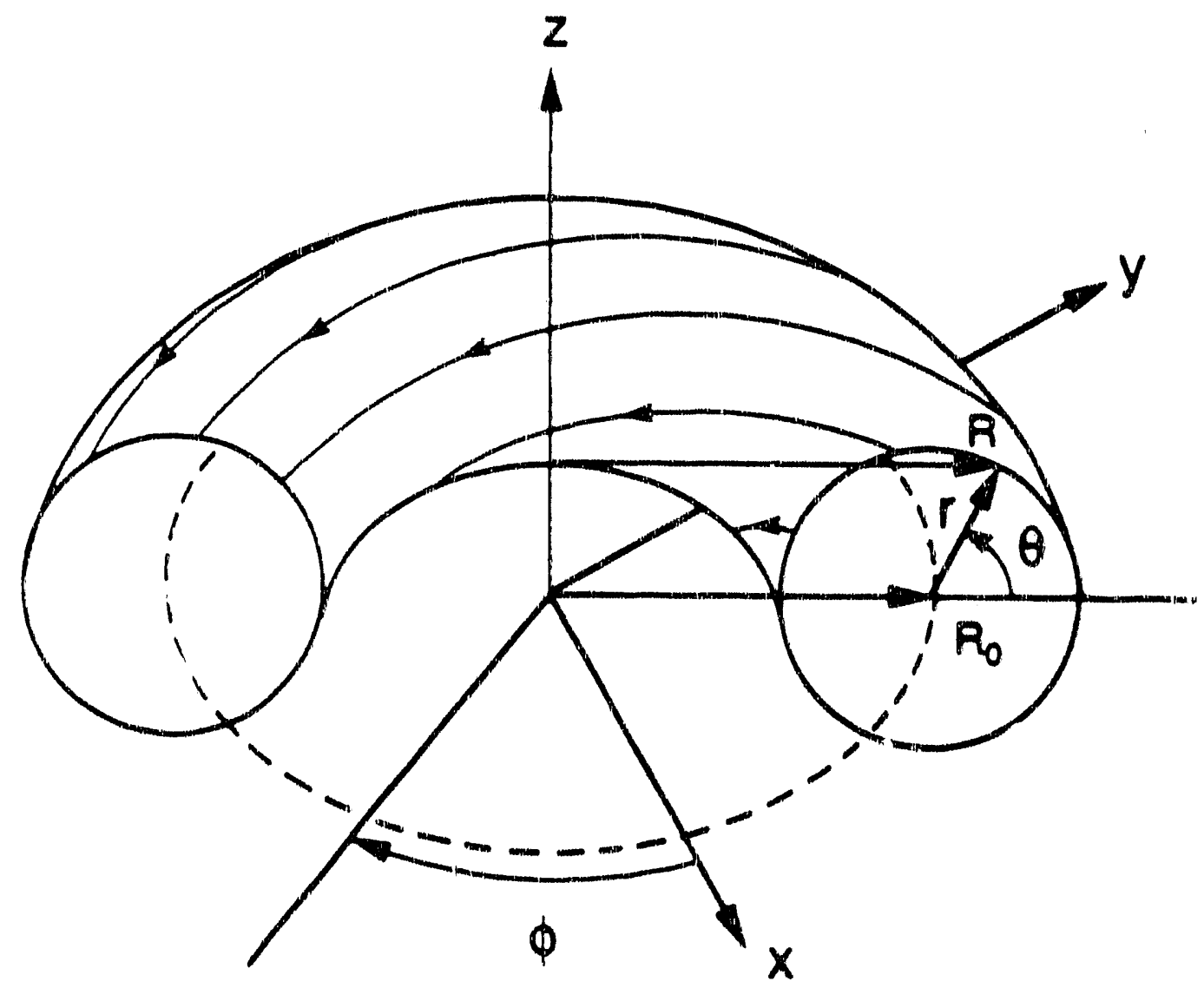

FLg, 3 


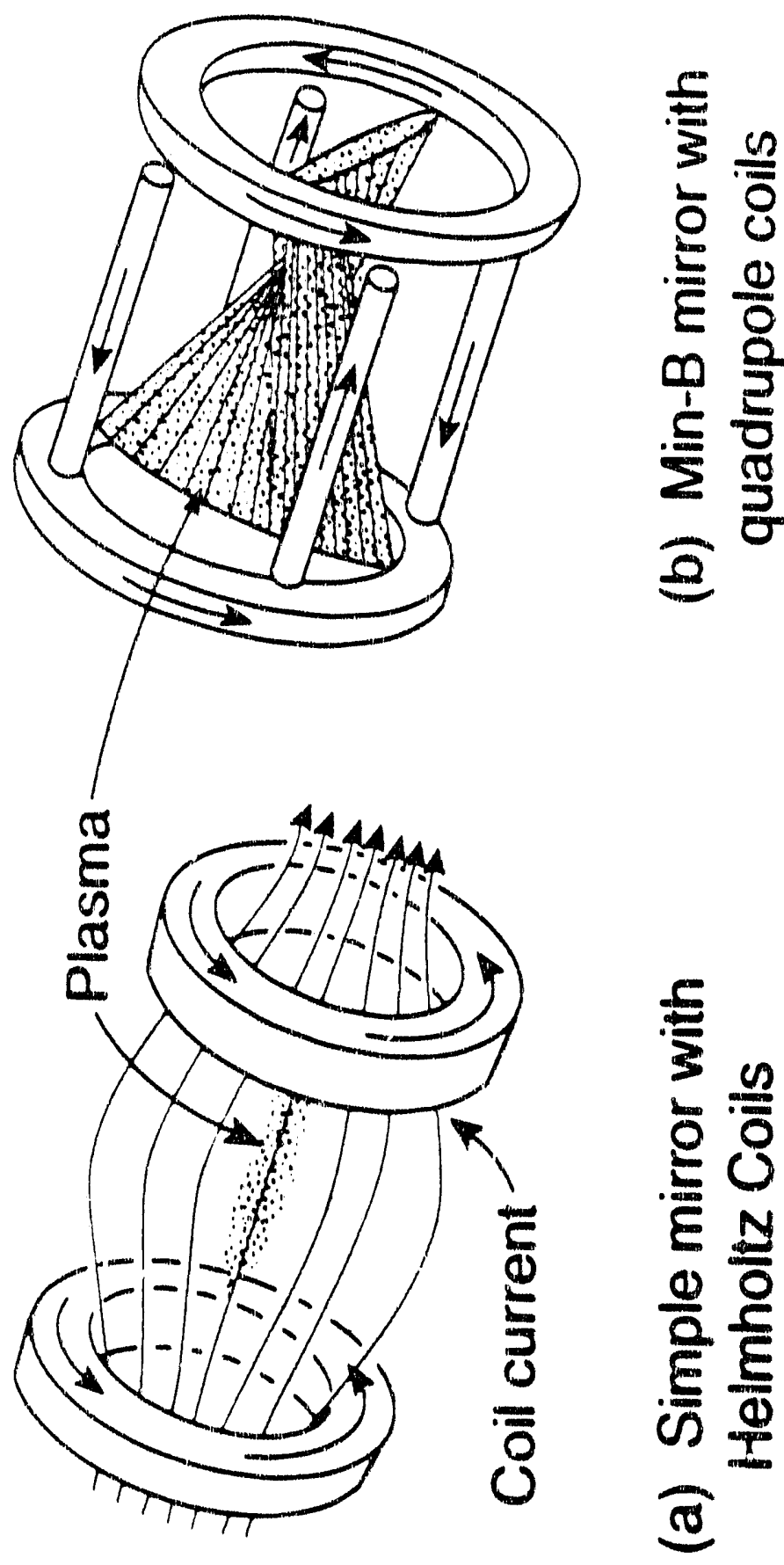

Fig. + 


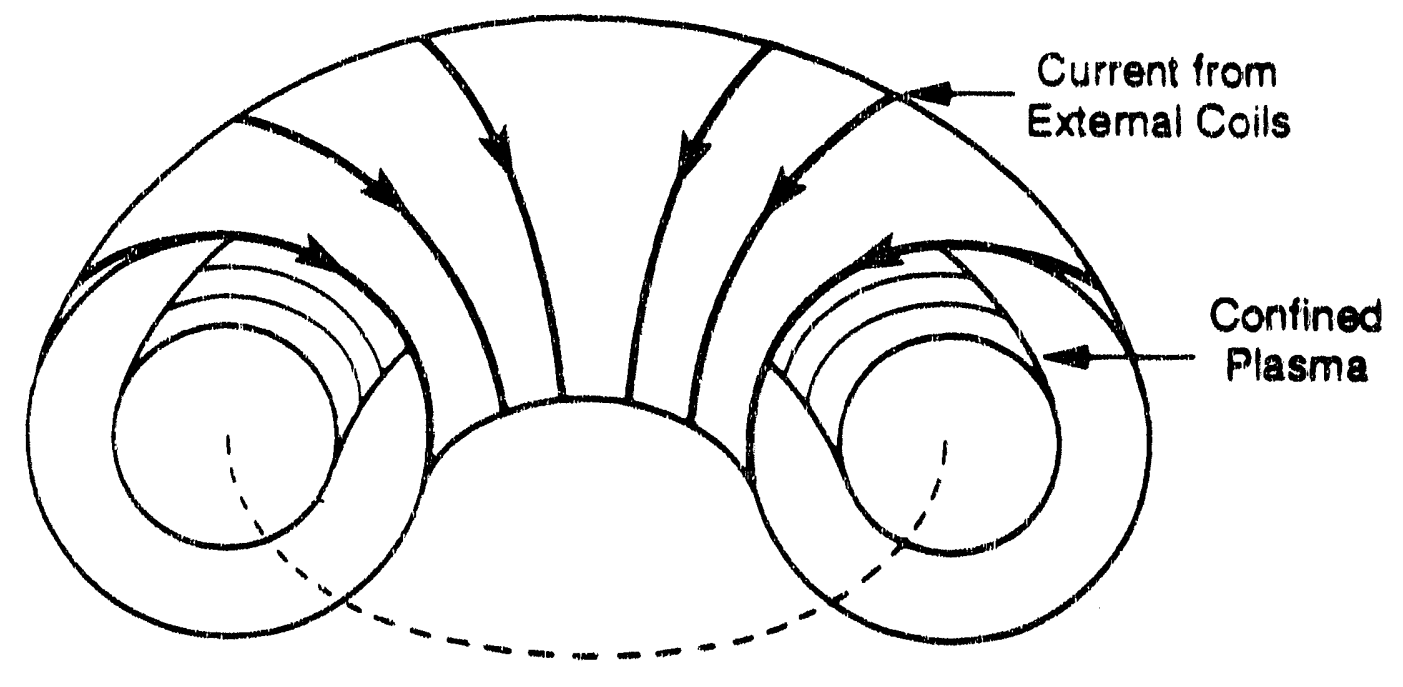

Fig. Ja 
th

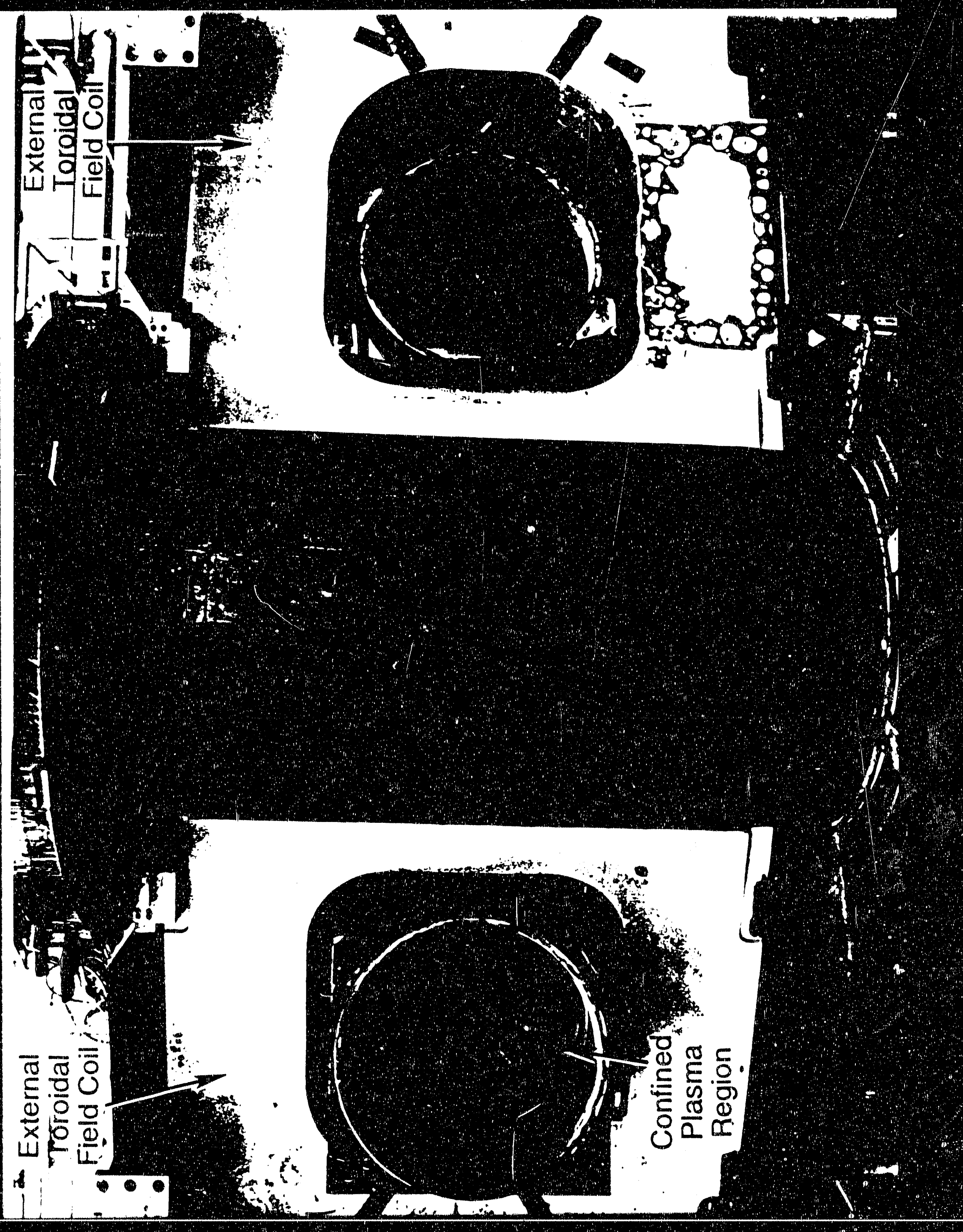




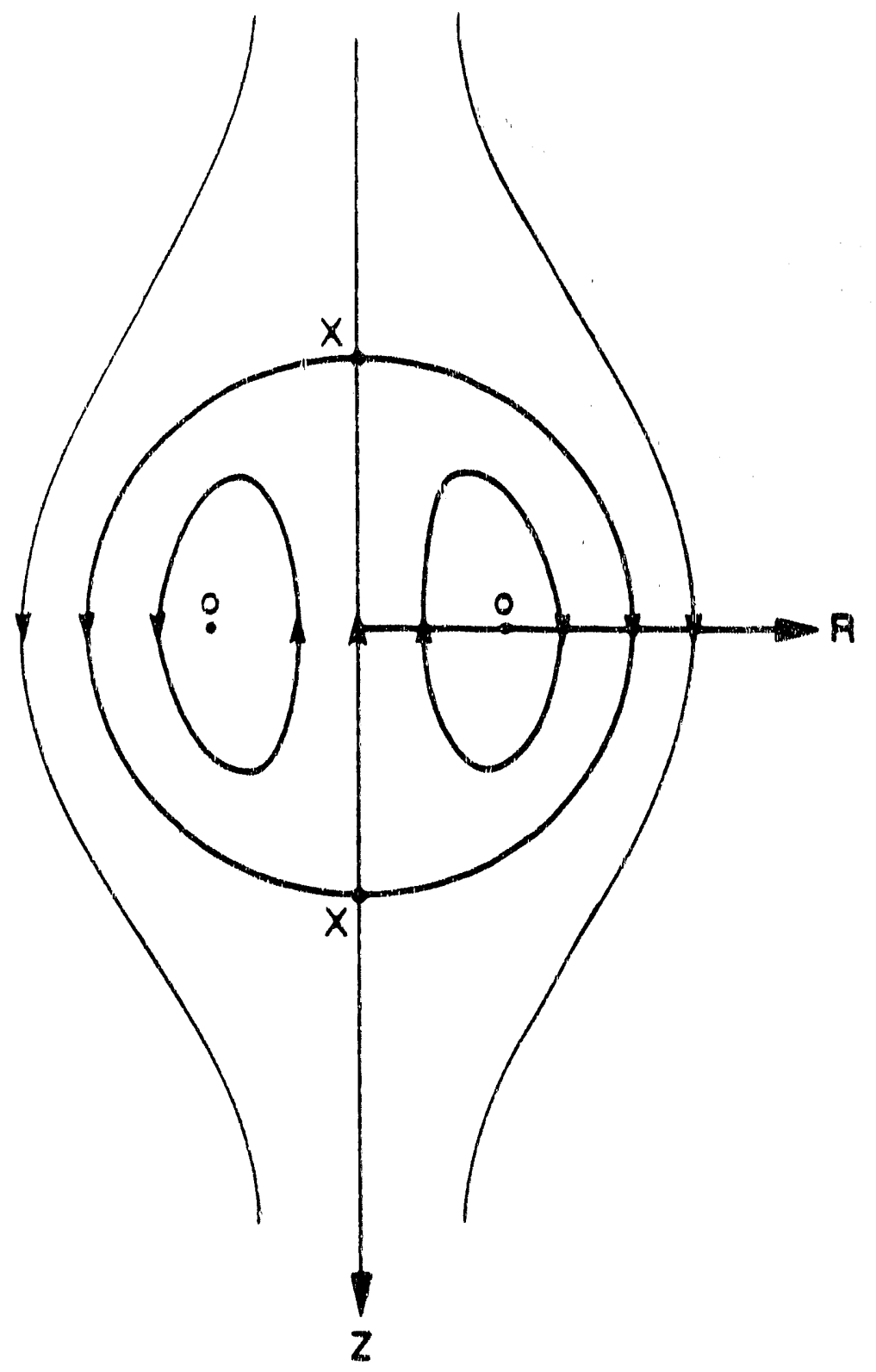

Fig. 0 


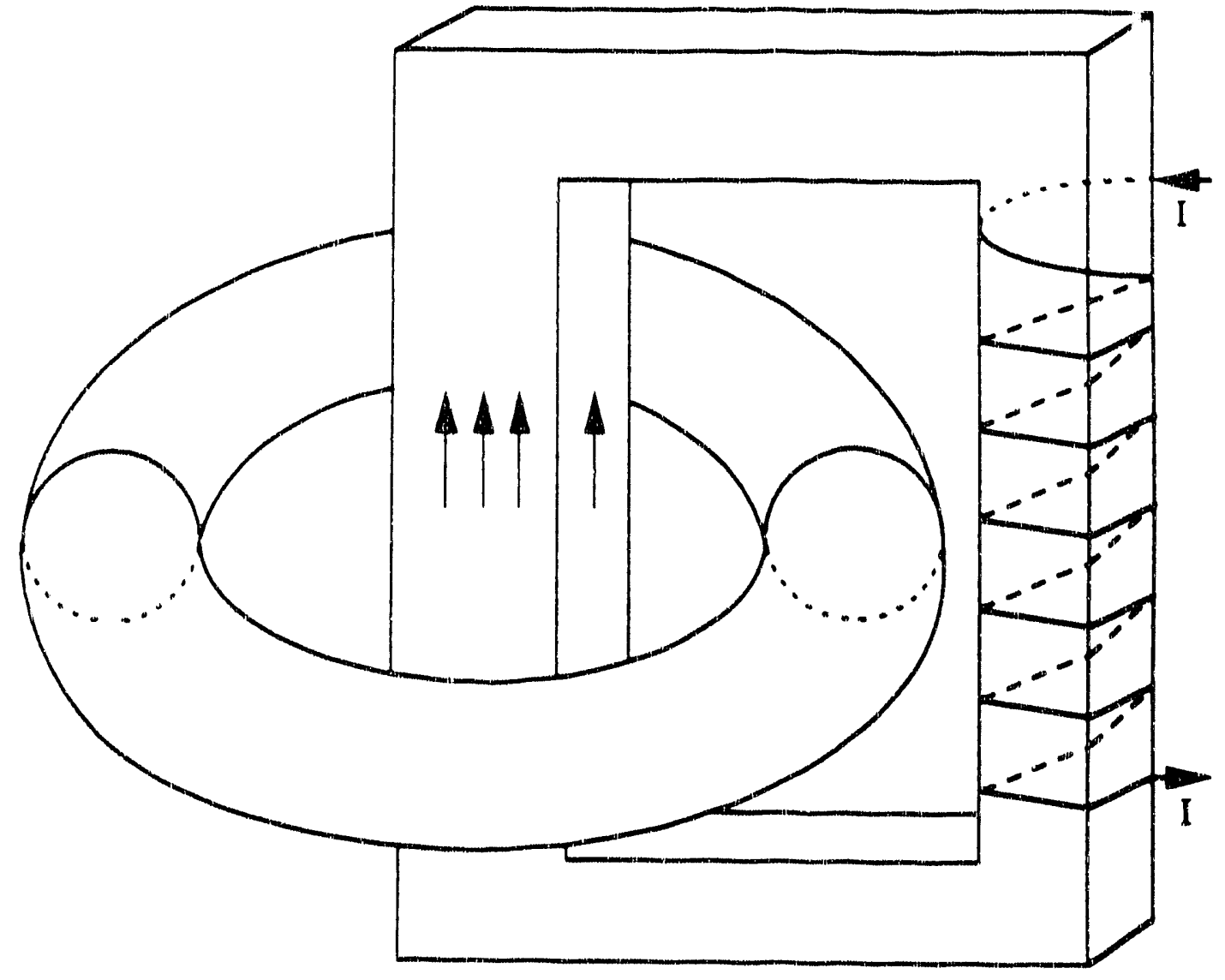

Fig. ? 


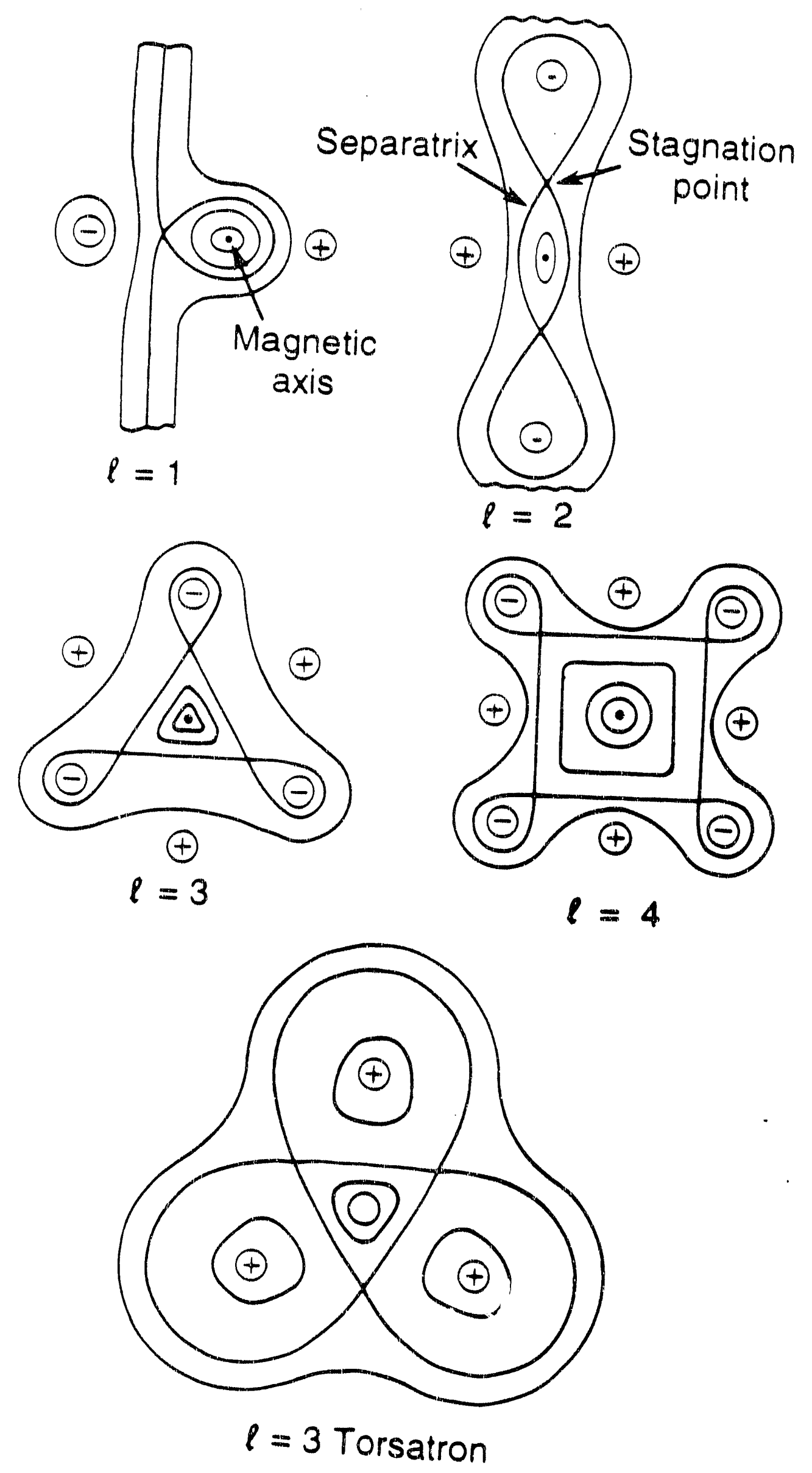

Fig. $8 a$ 


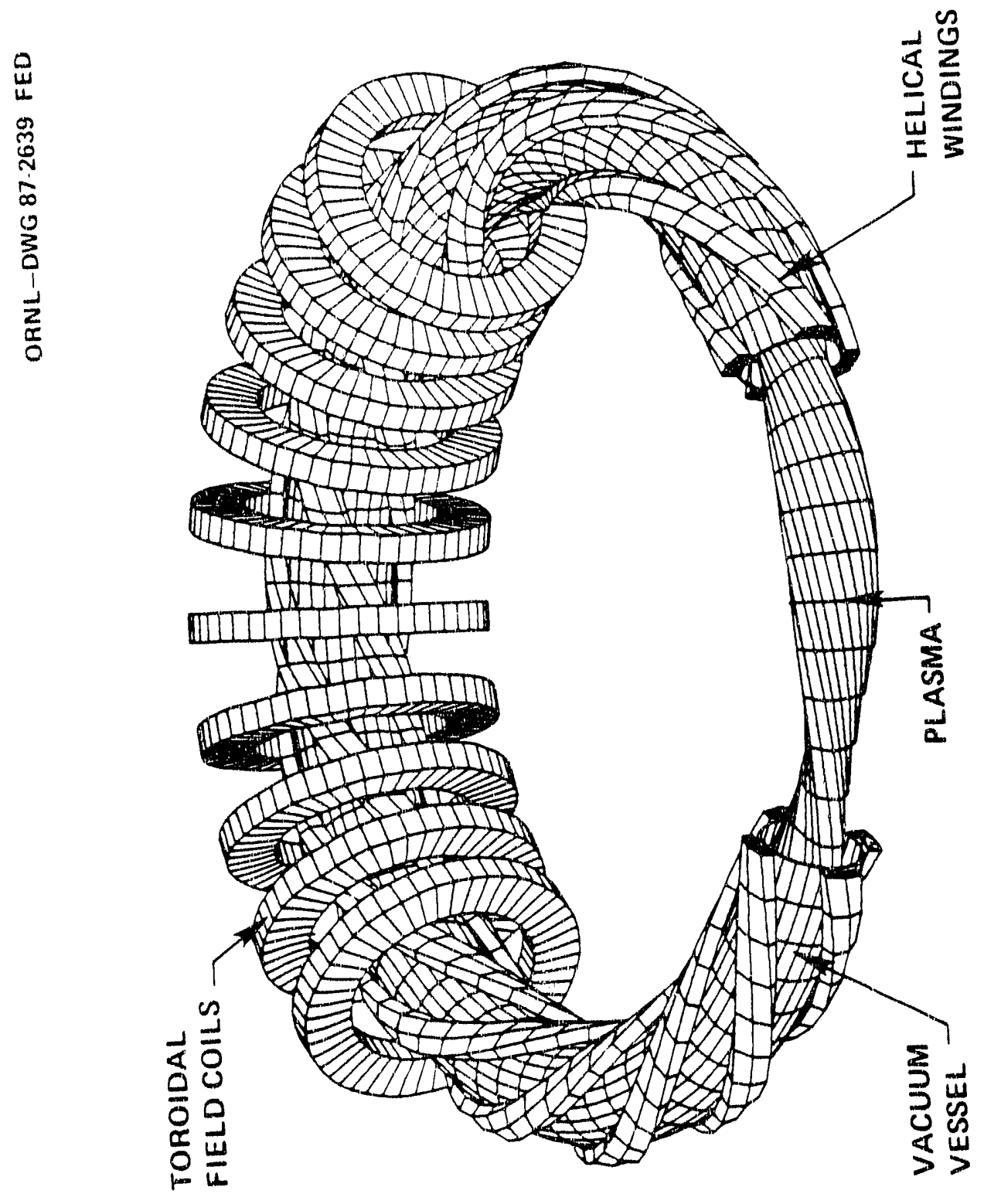

Fig. 8b 


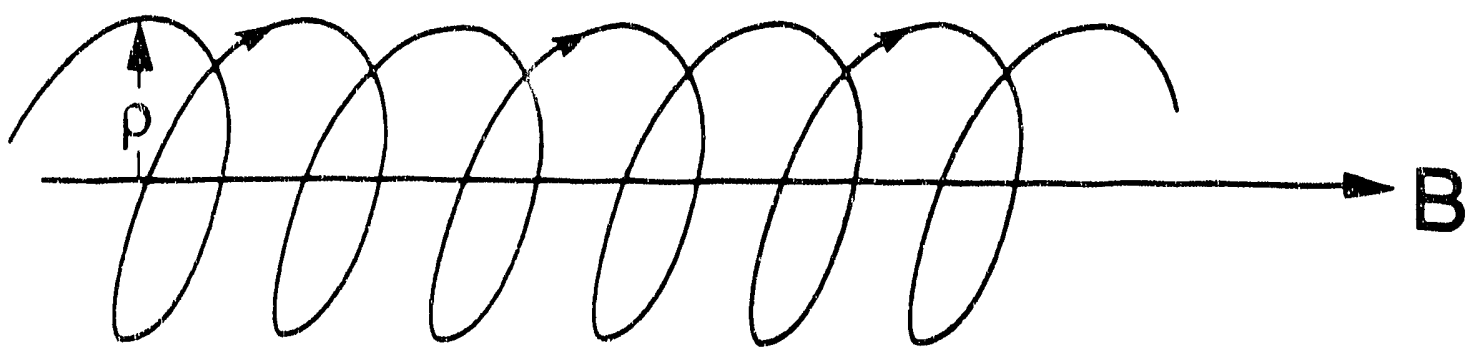

(a)

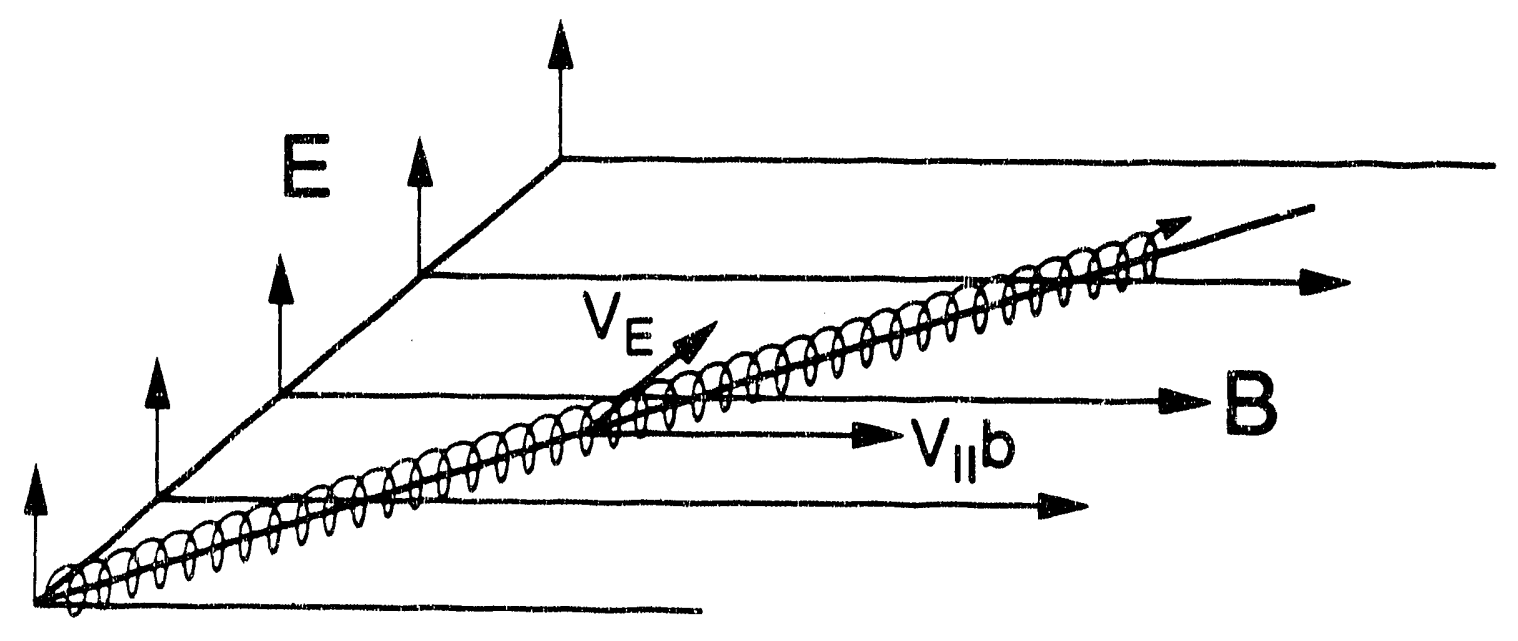

(b)

Fig. $y$ 


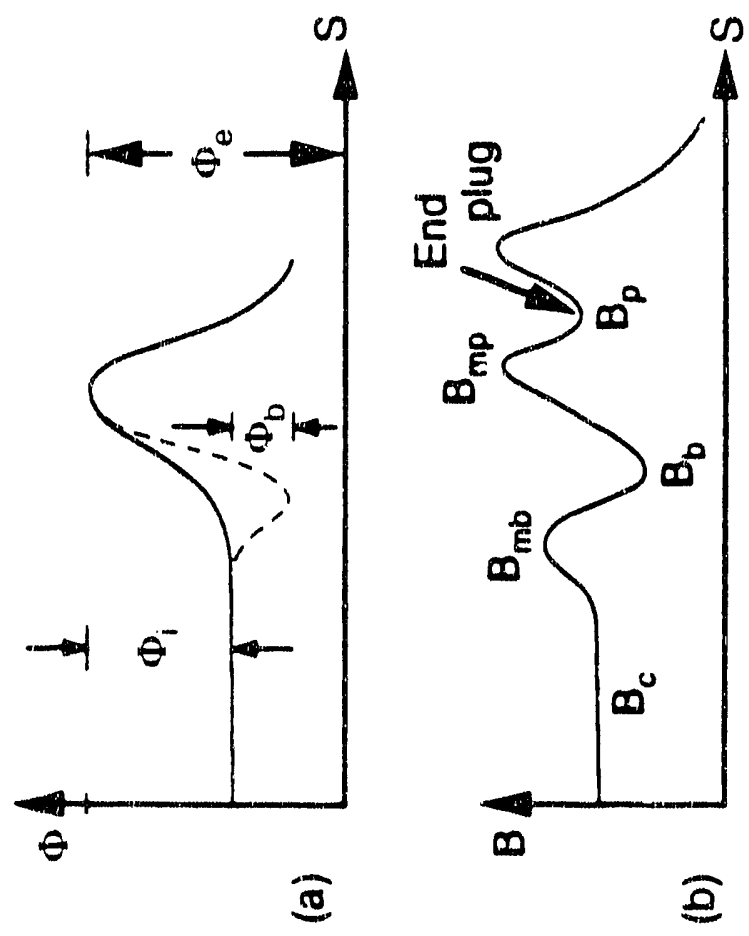

Fig. 10 


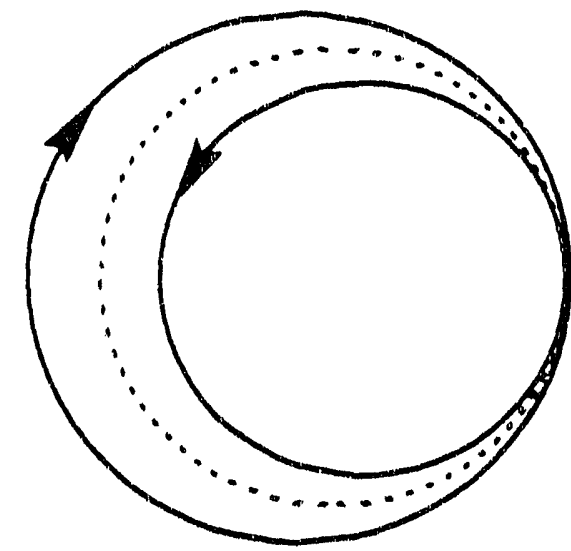

(a)

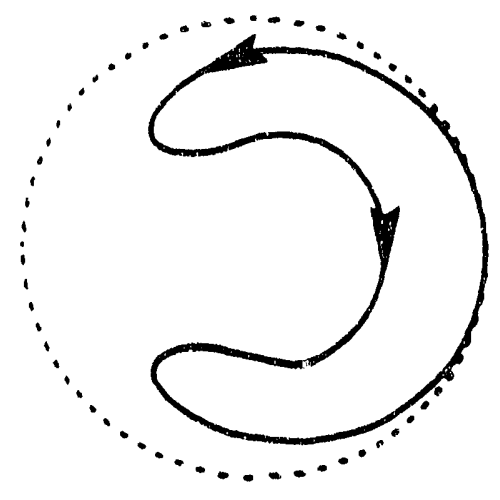

(b)

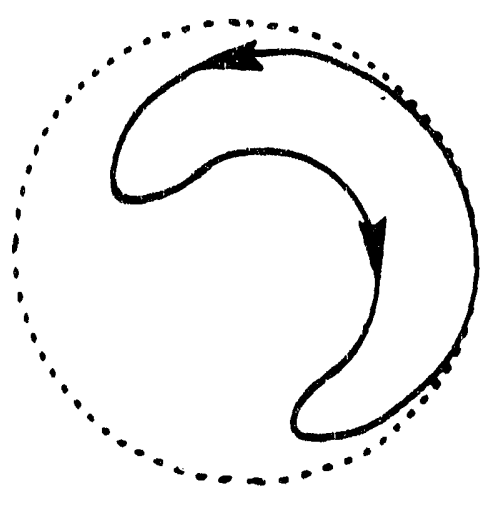

(c)

Fig. 11 


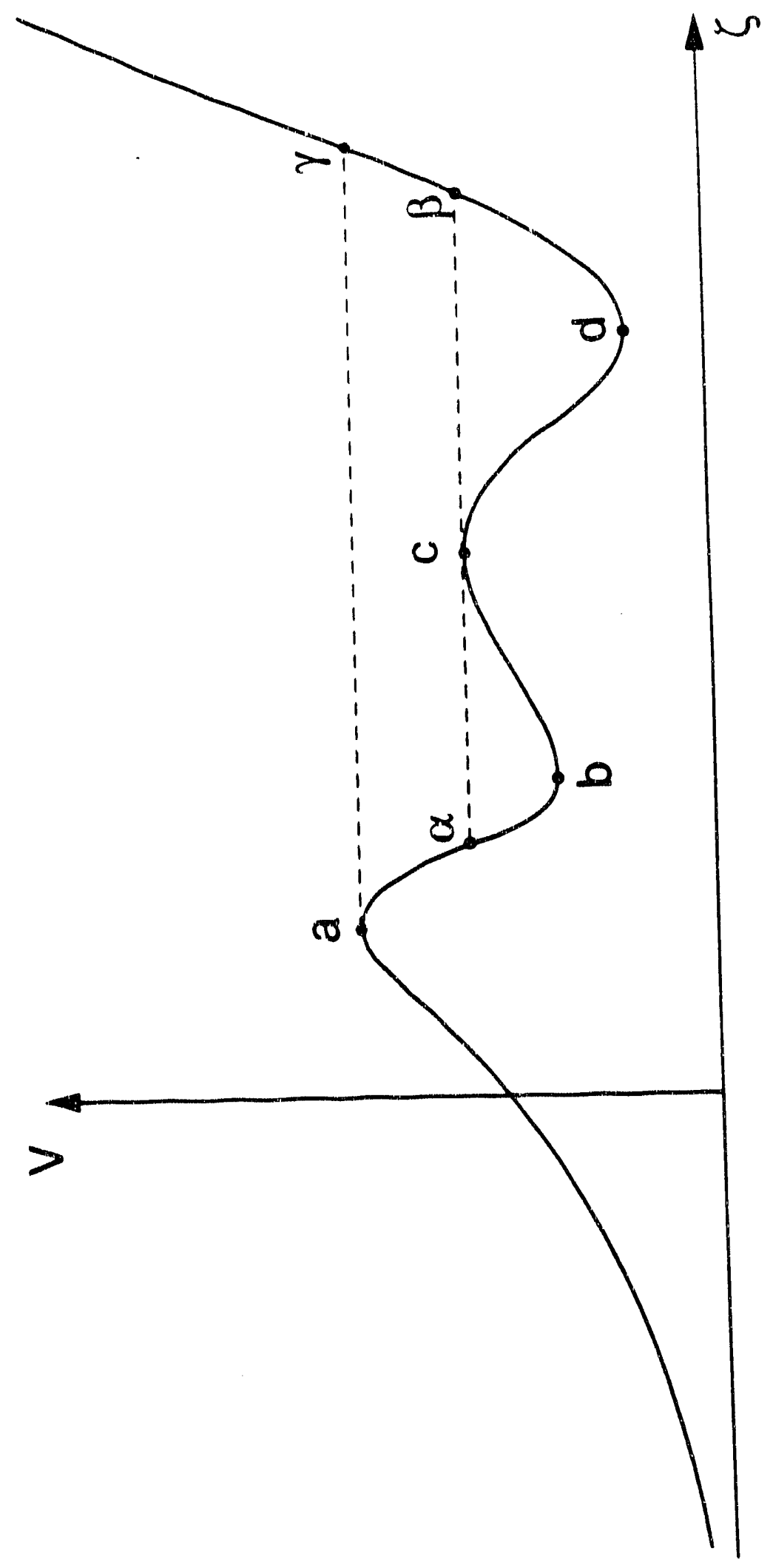

Fig. 12 


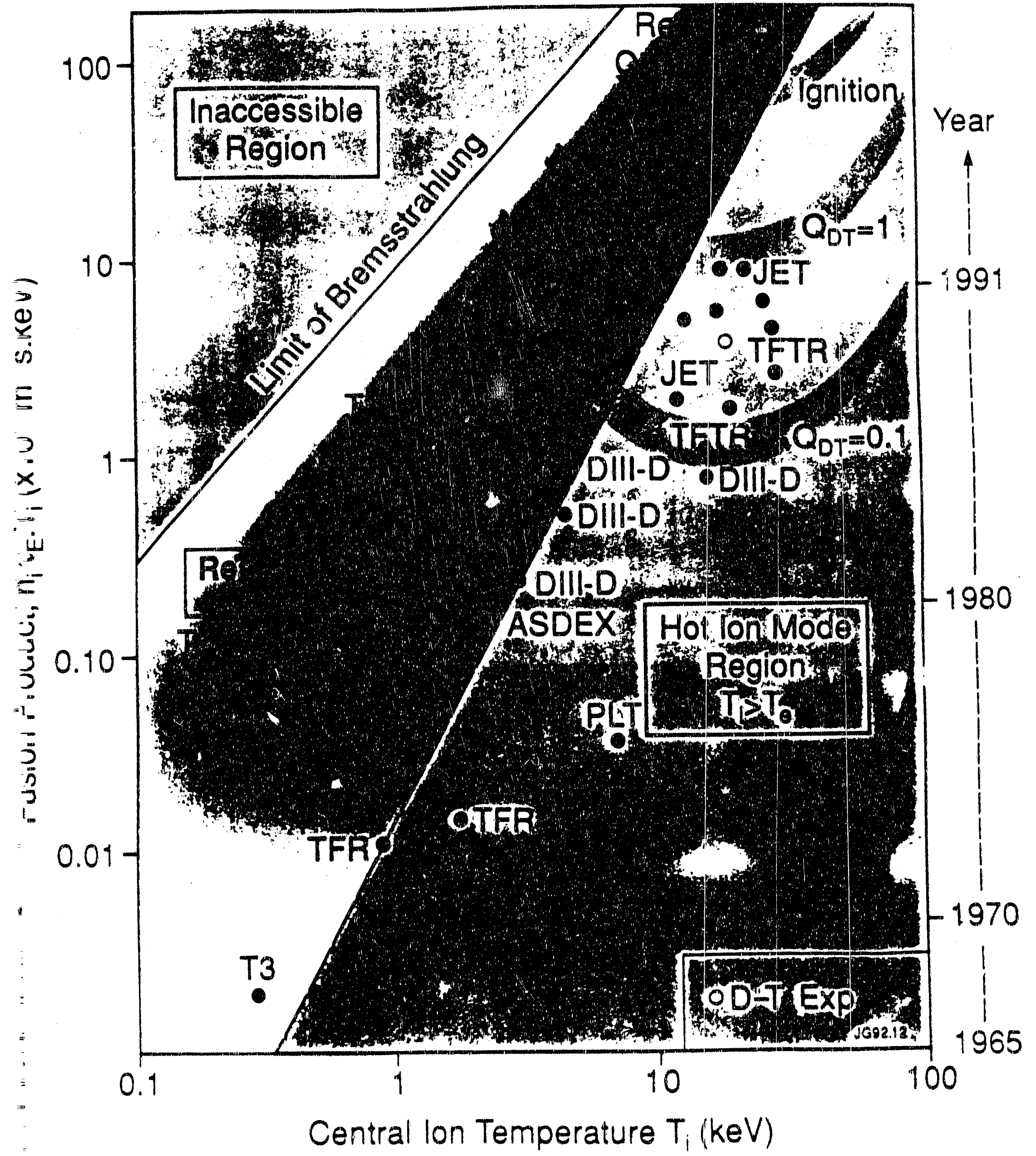



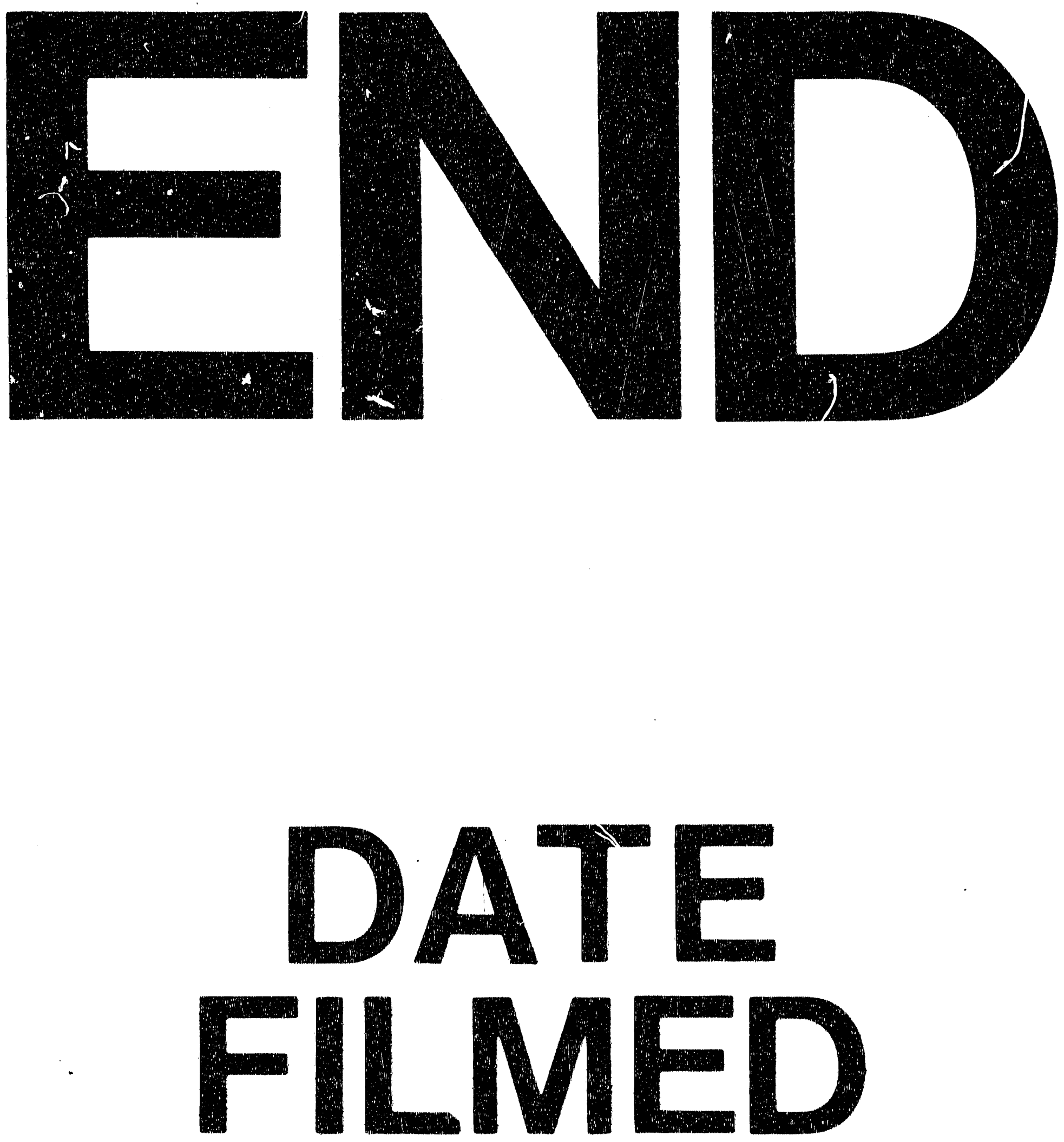

1
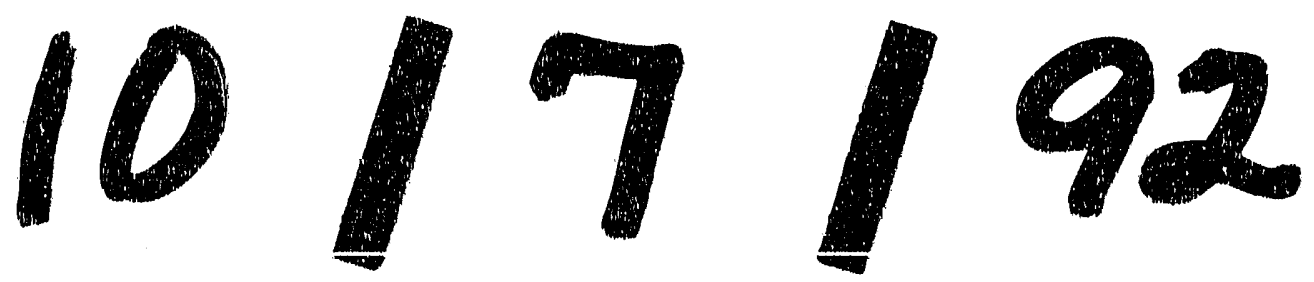
